\title{
A SNAI2-PEAK1-INHBA stromal axis drives progression and lapatinib resistance in HER2-positive breast cancer by supporting subpopulations of tumor cells positive for antiapoptotic and stress signaling markers
}

Sarkis Hamalian ${ }^{1}$, Robert Güth ${ }^{1}$, Farhana Runa ${ }^{1}$, Francesca Sanchez ${ }^{1}$, Eric Vickers ${ }^{1}$, Megan Agajanian ${ }^{1}$, Justin Molnar ${ }^{1}$, Tuan Nguyen ${ }^{1}$, Joshua Gamez ${ }^{1}$, Jonathan D. Humphries ${ }^{2,6}$, Anupma Nayak ${ }^{3}$, Martin J. Humphries ${ }^{2}$, Julia Tchou ${ }^{4}$, loannis K. Zervantonakis ${ }^{5}$ and Jonathan A. Kelber ${ }^{1 凶}$

(c) The Author(s) 2021

Intercellular mechanisms by which the stromal microenvironment contributes to solid tumor progression and targeted therapy resistance remain poorly understood, presenting significant clinical hurdles. PEAK1 (Pseudopodium-Enriched Atypical Kinase One) is an actin cytoskeleton- and focal adhesion-associated pseudokinase that promotes cell state plasticity and cancer metastasis by mediating growth factor-integrin signaling crosstalk. Here, we determined that stromal PEAK1 expression predicts poor outcomes in HER2-positive breast cancers high in SNAI2 expression and enriched for MSC content. Specifically, we identified that the fibroblastic stroma in HER2-positive breast cancer patient tissue stains positive for both nuclear SNAI2 and cytoplasmic PEAK1. Furthermore, mesenchymal stem cells (MSCs) and cancer-associated fibroblasts (CAFs) express high PEAK1 protein levels and potentiate tumorigenesis, lapatinib resistance and metastasis of HER2-positive breast cancer cells in a PEAK1-dependent manner. Analysis of PEAK1-dependent secreted factors from MSCs revealed INHBA/activin-A as a necessary factor in the conditioned media of PEAK1-expressing MSCs that promotes lapatinib resistance. Single-cell CyclF analysis of MSC-breast cancer cell co-cultures identified enrichment of $\mathrm{p}-\mathrm{Akt} \mathrm{t}^{\text {high }} / \mathrm{p}-\mathrm{gH} 2 \mathrm{AX}{ }^{\mathrm{low}}, \mathrm{MCL} 1^{\text {high }} / \mathrm{p}-\mathrm{gH} 2 \mathrm{AX}{ }^{\text {low }}$ and $\mathrm{GRP} 78^{\text {high }} / \mathrm{VIM}^{\text {high }}$ breast cancer cell subpopulations by the presence of PEAK1-expressing MSCs and lapatinib treatment. Bioinformatic analyses on a PEAK1-centric stroma-tumor cell gene set and follow-up immunostaining of co-cultures predict targeting antiapoptotic and stress pathways as a means to improve targeted therapy responses and patient outcomes in HER2-positive breast cancer and other stroma-rich malignancies. These data provide the first evidence that PEAK1 promotes tumorigenic phenotypes through a previously unrecognized SNAI2-PEAK1-INHBA stromal cell axis.

Oncogene (2021) 40:5224-5235; https://doi.org/10.1038/s41388-021-01906-2

\section{INTRODUCTION}

Cell state plasticity enhances intratumoral heterogeneity and has been shown to be a culprit underlying metastasis, therapy resistance and progression in cancer [1-4]. Previous studies have demonstrated a causative relationship between increased stromal tissue content (i.e., desmoplasia), including cancer-associated fibroblasts (CAFs) or mesenchymal stem cells (MSCs), in breast cancers and lapatinib resistance or metastasis [5-8]. In the case of HER2-positive breast cancer, where upregulation of the receptor tyrosine kinase HER2 (ErbB2) occurs in 20\% of all tumors [9], both trastuzumab- and lapatinib-based regimens offer significant clinical benefit [10]. However, a substantial percentage of these tumors display either primary resistance or may be initially sensitive but then adapt to develop acquired resistance [11], and clinical work suggests that patients who progress on lapatinib therapy commonly develop metastatic disease [12]. While recent work has reported that stromal fibroblasts limit HER2 kinase therapy responses via antiapoptotic signaling [13], the stromal cell non-autonomous mechanisms underlying HER2-targeted therapy resistance and/or resistance-associated metastasis remain poorly understood.

Pseudopodium-Enriched Atypical Kinase One (PEAK1 or SGK269) is a cytoskeleton-associated pseudokinase [14] and member of the new NKF3 kinase family that has been

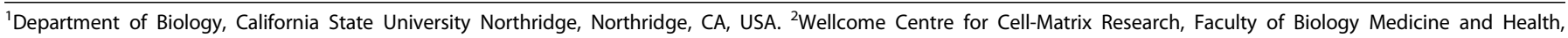

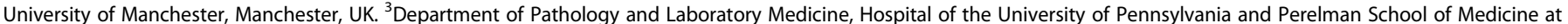

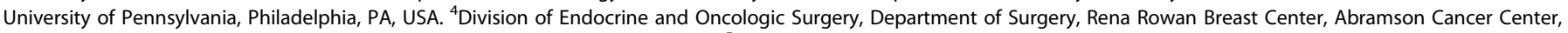

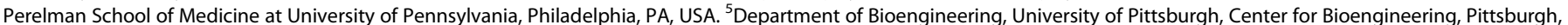
PA, USA. ${ }^{6}$ Present address: Department of Life Sciences, Manchester Metropolitan University, Manchester, UK. ${ }^{\bowtie}$ email: jonathan.kelber@csun.edu 
demonstrated to play key cancer cell autonomous roles in cancer initiation and progression across multiple cancer types including breast [15-17], pancreatic [14, 18], lung [19] and colon [14, 20, 21]. We previously reported that PEAK 1 functions downstream of elF5A1/2-dependent translation in mediating epithelialmesenchymal transition (EMT), metastasis and transforming growth factor beta (TGF $\beta$ )/fibronectin signaling [15-17, 22]. In this regard, PEAK1 has been identified as part of the metaadhesome [23] and core constituent of the fibroblast adhesome [24]. Zheng and colleagues reported that PEAK1 is a critical adapter protein governing Shc1 association with cytoskeletal reorganization, trafficking and signal termination proteins downstream of EGF/Akt/PTPN12 activity to mediate cell invasion [25].

Here, we address whether PEAK1 may promote tumorigenesis via the non-epithelial stromal compartment of solid tumors. To this end, we report that PEAK1 expression in breast cancer stroma is associated with relapse in HER2-positive breast cancer and that PEAK1 is predominantly expressed in tumor associated SNAI2positive fibroblast-like cells. In agreement with these data, patientderived CAFs and MSCs express PEAK 1 and can promote malignant phenotypes and lapatinib resistance in vitro and in vivo in a PEAK1dependent manner. Finally, we combine protein array and singlecell CyclF multiplex methods to identify a previously unrecognized PEAK1-INHBA-antiapoptotic stromal-tumor cell signaling axis that may be leveraged to abrogate therapeutic resistance in HER2positive breast cancer and improve patient outcomes.

\section{MATERIALS AND METHODS}

\section{Cell culture}

Cell origin and culture method details are described in the Supplementary Materials and Methods.

\section{Bioinformatics}

Data mining and analyses procedures are described in the Supplementary Materials and Methods.

\section{Immunohistochemistry}

Formalin-fixed tissue samples were sent to the UCLA Tissue Procurement Core Laboratory for paraffin embedding, tissue sectioning and H\&E staining. Alternatively, primary breast cancer tissue was obtained as single tumor sections from Dr. Julia Tchou or purchased as a tissue microarray (TMA) from USBiomax. Details on antibody, staining and imaging procedures are described in the Supplementary Materials and Methods.

\section{Conditioned media (CM)}

Cells were plated in $10 \mathrm{~cm}$ plates at $6 \mathrm{e} 5$ cells and incubated until $70 \%$ confluent. Media in each $10 \mathrm{~cm}$ plate was changed to $6 \mathrm{~mL}$ of appropriate media without serum and incubated for an additional $48 \mathrm{~h}$. Mock/control media was made by placing the same media into a plate without cells for $48 \mathrm{~h}$. Media was collected, centrifuged at $1000 \mathrm{rpm}$ for $5 \mathrm{~min}$ and used right away or stored at $-80^{\circ} \mathrm{C}$ in $15 \mathrm{~mL}$ aliquots until needed. Before the CM was used in experiments, it was diluted 1:1 with the appropriate fresh serum-free media. Subsequent ELISA analyses on CM for activin-A was performed using the activin-A DuoSet ELISA (R\&D Systems) in accordance with manufacturers instructions.

\section{Western blot}

Cell extract collection and immunoblot reagents and procedures are described in the Supplementary Materials and Methods.

\section{Immunocytochemistry}

Details on antibody, staining and imaging procedures are described in the Supplementary Materials and Methods.

\section{Chorioallantoic membrane assay (CAM assay)}

Animal origin and use procedures are described in the Supplementary Materials and Methods. All procedures were completed in accordance with
IACUC protocol \# 1920-008b. qPCR analysis details are also described within the Supplementary Materials and Methods.

\section{Lentiviral transduction}

Cells were plated at $4.8 \mathrm{e} 6$ cells/well into a 6 well plate and left to attach overnight. Viral mixes were created with an aliquot of virus into complete media and polybrene at $8 \mu \mathrm{g} / \mathrm{ml}$ (Sigma-Aldrich) to have a target multiplicity of infection (MOI) of 5 . Viral particles contained a puromycin resistant pKLO.1 vector with a scramble shRNA or PEAK1-specific shRNA (5 different constructs). Viral mixes were added to their respective wells and left to incubate for $24 \mathrm{~h}$, after which regular media was replaced. The following day, media was changed and supplemented with $1 \mathrm{ug} / \mathrm{mL}$ puromycin. Cells were expanded and knockdown efficacy was validated by Western blotting.

\section{Cell proliferation/viability assay}

Cell proliferation/viability was measured using the CellTiter $96^{\circledR}$ AQueous One Solution (Promega). Cell plating and analysis procedures are described in the Supplementary Materials and Methods.

\section{IncuCyte}

IncuCyte ${ }^{\circledast}$ Live Cell Analysis Imaging System was used according to manufacturer's protocol. Cell plating and analysis procedures are described in the Supplementary Materials and Methods.

\section{Protein microarray}

The semi-quantitative RayBio L-series mouse antibody array L-308 was used according to manufacturer protocol for analysis of cell lysates from the $\mathrm{C} 3 \mathrm{H} 10 \mathrm{~T} 1 / 2$ shRNA derivatives. Processed slides were imaged and analyzed using a GenePix 400B instrument and Molecular Devices software.

\section{Cyclic immunofluorescence (CyclF)}

Cyclic multiplex antigen staining and Hoescht nuclear counterstaining was carried out on paraformaldehyde (PFA)-fixed mono- or co-culture cells on ultra-optically clear, flat-bottom, black-walled 96-well plates as previously described [26]. Cell plating, reagent, staining and analysis procedures are described in the Supplementary Materials and Methods.

\section{Statistics}

All quantified data were plotted and analyzed in GraphPad Prism with ANOVA, Student's $t$ test, or nonlinear regression analysis. Data reported are representative of at least 3 independent biological replicates and are reported as technical replicate averages \pm SEM, unless otherwise indicated. $* *^{* *}, * * *$ or $* * * *$ represent $p$ values $<0.05,0.01,0.001$, or 0.0001 respectively, unless otherwise noted.

\section{RESULTS}

\section{A SNAI2-PEAK1 stromal axis correlates with disease} progression in HER2-positive breast cancer

We first examined patient survival across all breast cancer subtypes in relation to PEAK1 expression levels. The KMPlot resource enabled assessment of relapse-free survival (RFS), distant metastasis-free survival (DMFS) and overall survival (OS) across more than 3000 patients [27, 28]. Elevated PEAK1 expression across all breast cancer subtypes predicted a significant, though slight, increase in RFS (Fig. 1a), while elevated PEAK1 expression in HER2-positive breast cancers correlated with decreased RFS suggesting a role for PEAK1 in this more aggressive breast cancer subtype (Fig. 1b). In contrast, elevated PEAK1 expression alone across all breast cancer subtypes had a very modest prognostic association with OS or DMFS (Supplementary Fig. 1). In parallel, we mined data [29] on breast cancer stromal gene expression and discovered that PEAK1 expression was significantly higher in malignant breast stroma (Fig. 1c), and that elevated stromal PEAK1 expression positively correlated with disease relapse (Fig. 1d).

To identify breast cancer stromal gene networks associated with increased PEAK1 expression and poor outcome, we analyzed the relationships between expression patterns for 

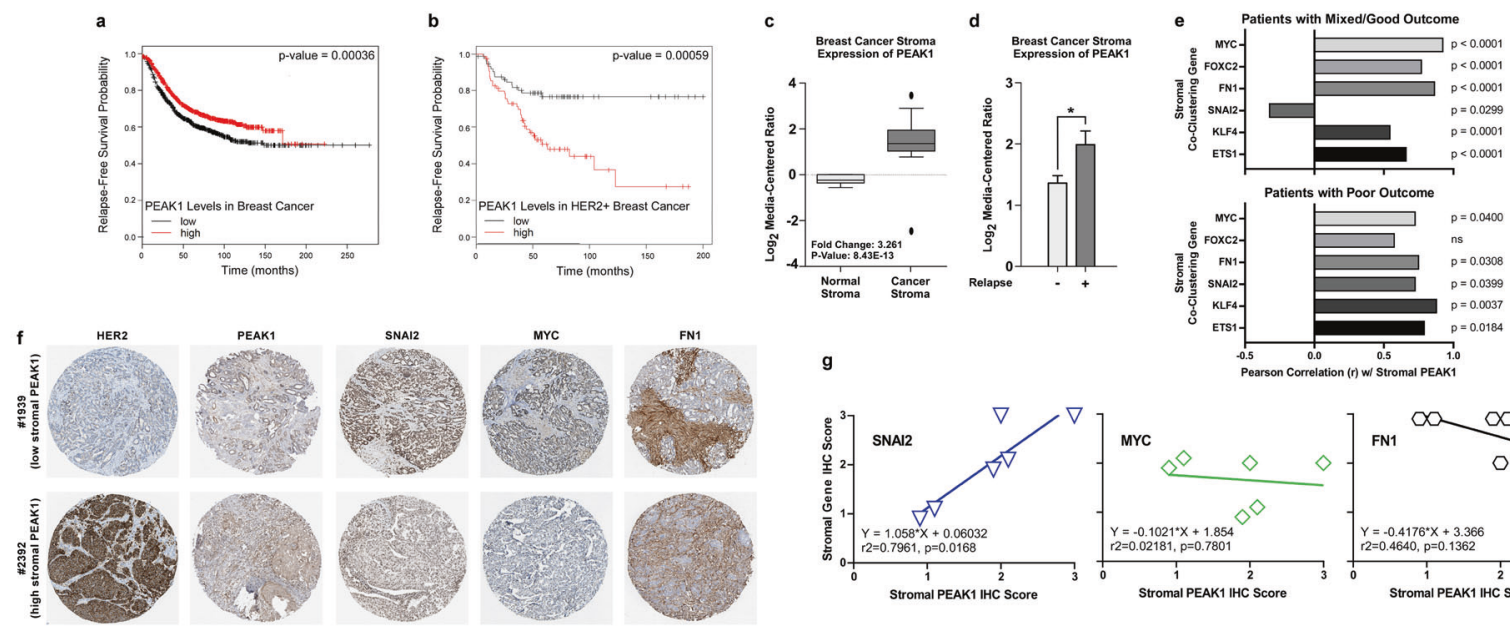

g
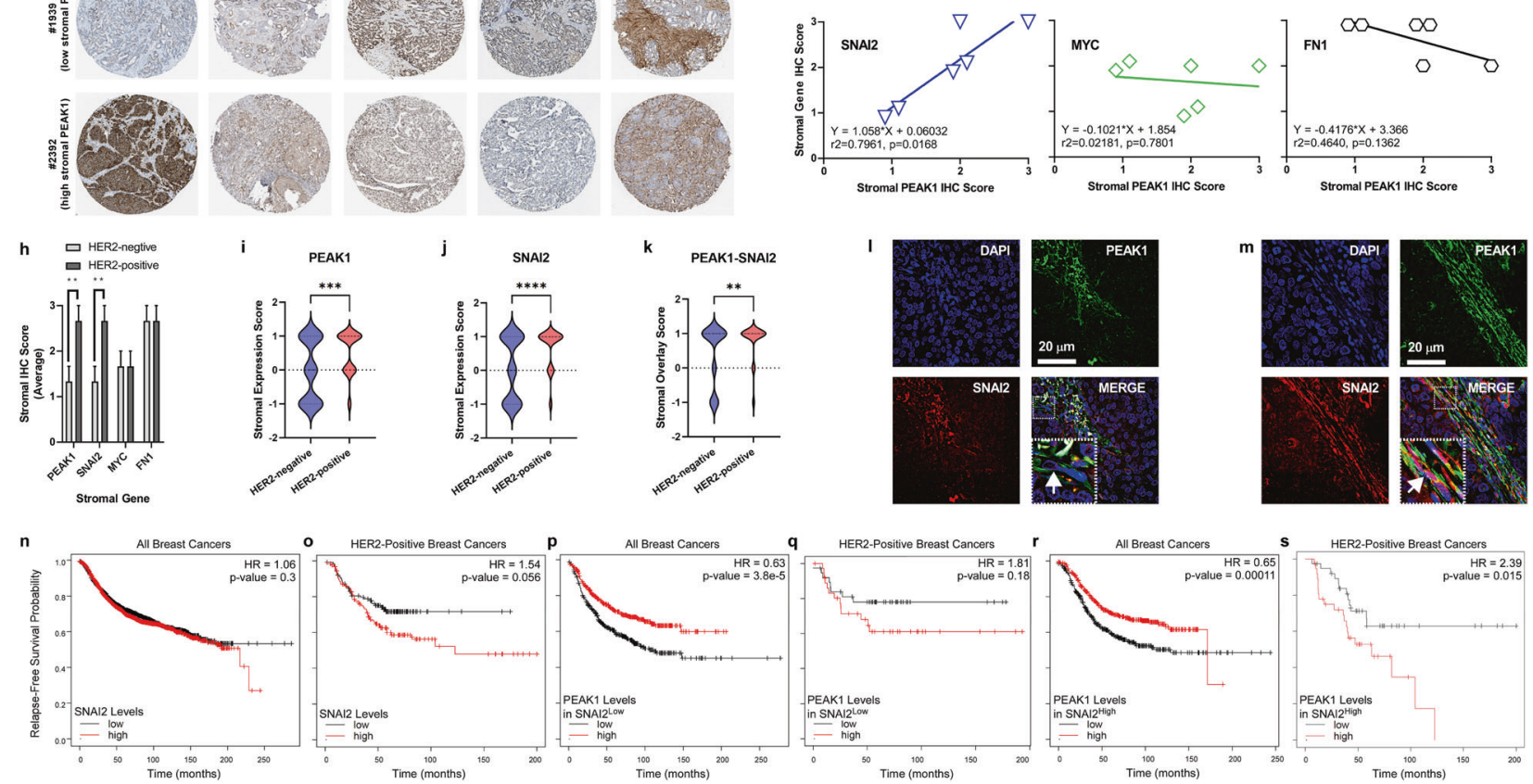

Fig. 1 A SNAI2-PEAK1 axis correlates with disease relapse and co-stains the non-epithelial fibroblastic stroma in HER2-positive breast cancer. a Kaplan-Meier relapse-free survival (RFS) curves for patients with low or high PEAK1 transcript levels across all breast cancer subtypes $(n=1784)$. b Kaplan-Meier RFS curves for HER2-positive breast cancer patients with low or high PEAK1 transcript levels $(n=272)$. c PEAK1 transcript levels in normal breast stroma and breast cancer stroma across all subtypes $(n=6$ and 53, respectively). d PEAK1 transcript levels in breast cancer stroma of relapse-free patients and those with disease recurrence across all subtypes $(n=42$ and 11 , respectively). e Expression correlation analysis of PEAK1 and ETS1, KLF4, SNAI2, FN1, FOXC2 or MYC in patients having mixed or good outcomes (top, $n=45$ ) and patients having poor outcomes (bottom, $n=8$ ). $\mathbf{f}$ Representative IHC images for HER2, PEAK1, SNAI2, MYC and FN1 in breast cancer tissue where PEAK1 expression in the stromal compartment is low (top) or high (bottom). $\mathbf{g}$ Stromal IHC score correlation and linear regression analyses of PEAK1 and SNAI2, MYC or FN1 across six breast cancer tissue samples (patient \#s 1874, 1939, 2428, 1920, 2392 and 3257). h Average stromal IHC scores for PEAK1, SNAI2, MYC and FN1 in three HER2-negative patients and three HER2-positive patients. i-k Violin plots of quantified PEAK1, SNAI2 or PEAK1/SNAI2 stromal expression from IHC/IF data on a 144-breast cancer sample tissue microarray (79 HER2-positive cases). $\mathbf{j}-\mathbf{m}$ Representative 3D deconvolution widefield microscopy images for PEAK1 and SNAI2 in TB130 (HER2-negative) and TB122 (HER2-positive) breast cancer tissues. n-o Kaplan-Meier relapse-free survival (RFS) curves for patients with low or high SNAI2 in all breast cancer subtypes ( $n, n=1784)$ or HER2-positive breast cancer $(\mathbf{o}, n=272)$. Kaplan-Meier relapse-free survival (RFS) curves for patients with low or high PEAK1 in all SNAI2 ${ }^{\text {low }}$ or SNAI2 $2^{\text {high }}$ breast cancers ( $\mathbf{p}$ and $\left.\mathbf{r}, n=892\right)$ and HER2-positive SNAI2 ${ }^{\text {low }}$ or SNAI2 ${ }^{\text {high }}$ breast cancers $(\mathbf{q}$ and $\mathbf{s}$, $n=136) .{ }^{*}, * * * * *$ and ${ }^{* * * *}$ indicates $p$ value $<0.05,0.01,0.001$ and 0.0001 , respectively, as determined by a Student's $T$ test.

gene signatures corresponding to epithelial (9 genes), mesenchymal (19 genes), stem (4 genes) and mesenchymal stem (15 genes) markers. These signatures were clustered relative to PEAK1 in stromal tissue samples across patient groups previously classified as having poor, mixed or good outcomes [29] (Supplementary Fig. 2a). Expression correlation analysis identified six genes (i.e., ETS1, KLF4, SNAI2, FN1, FOXC2 and $\mathrm{MYC}$ ) that strongly clustered with PEAK1 in the poor outcome group. Notably, the SNAI2-PEAK1 relationship shifted from a significant negative correlation in patients having mixed/good outcomes to a significant positive correlation across patients having poor outcomes (Fig. 1e). In further support of a SNAI2-PEAK1 stromal cell signaling axis in breast cancer, we noted a significant positive correlation between SNAI2 and PEAK1 protein levels in the stromal compartment of breast cancer samples (Fig. 1f, g), and that these SNAI2 and PEAK1 protein levels were specifically expressed at higher levels within the stroma of HER2-positive tumors (Fig. 1h). We further stained tissue microarrays (TMAs) for PEAK1, SNAI2 and $\mathrm{CDH} 1$ and analyzed the PEAK1/SNAI2 expression/co-expression patterns within the $\mathrm{CDH} 1$-negative stromal compartment. This revealed that the stromal co-expression of PEAK1 and SNAI2 was significantly increased in HER2-positive tumor tissues $(n=$ 79) (Fig. $1 \mathrm{i}-\mathrm{k}$ and Supplementary Fig. 2b). Additional patient sample staining revealed further that the cytoplasmic PEAK1 and nuclear SNAI2 expression within the stromal tissue of HER2-positive breast cancers can occur within the same fibroblastic cell types - a pattern not observed in the HER2negative patient samples (Fig. 1l, m). Like PEAK1, elevated SNAI2 expression in HER2-positive breast cancer predicts 
a $\quad$ b

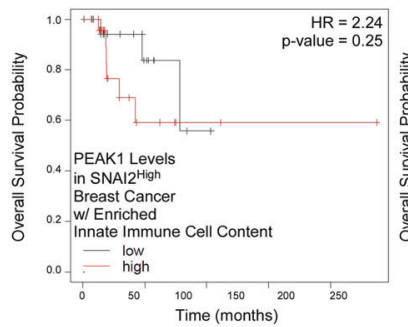

e

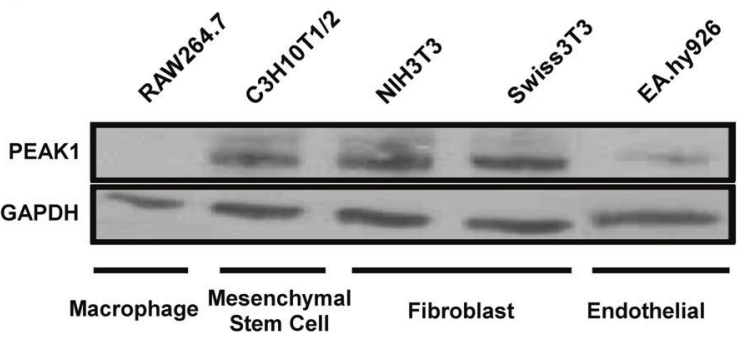

g

Collagen

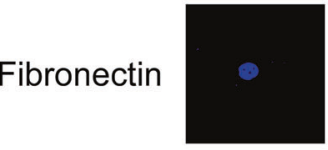

Laminin

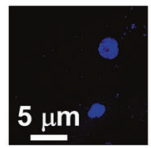

h

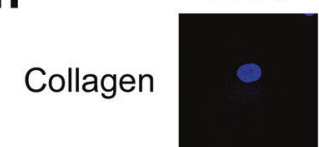

Fibronectin

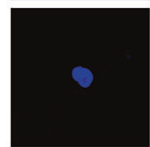

Laminin
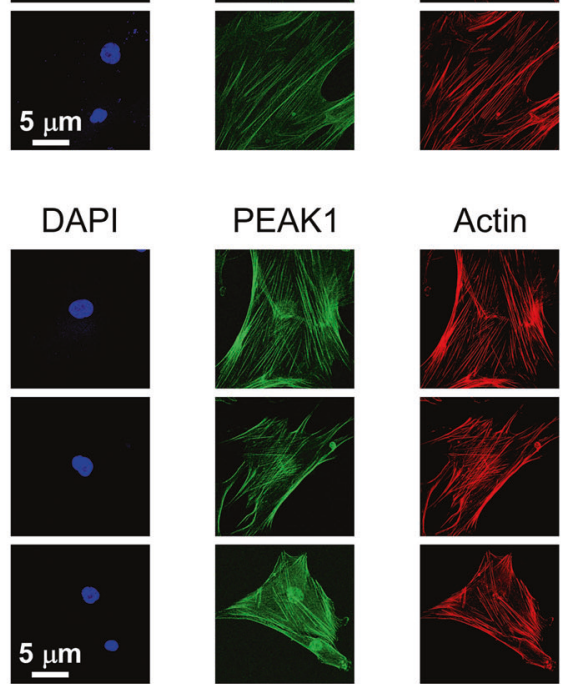

c

C
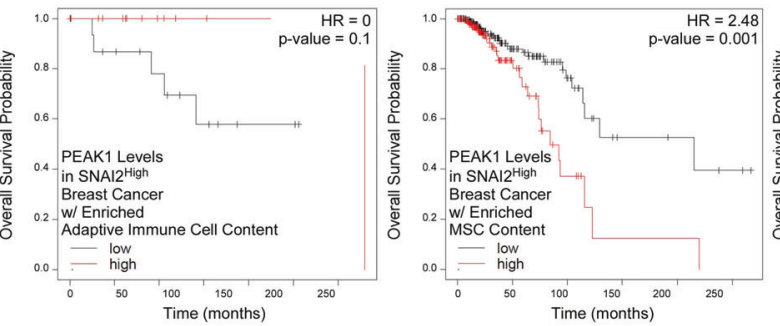

d

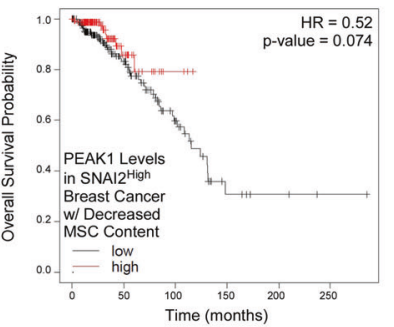

f

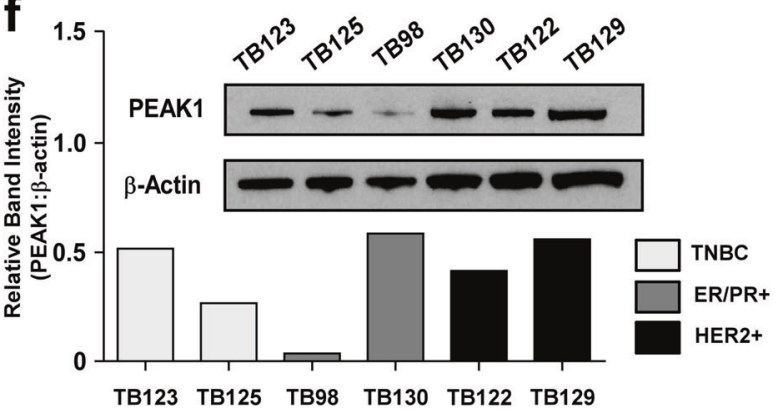

$\begin{array}{lllll}\text { TB123 } & \text { TB125 } & \text { TB98 } & \text { TB130 } & \text { TB122 } \\ \text { TB129 }\end{array}$
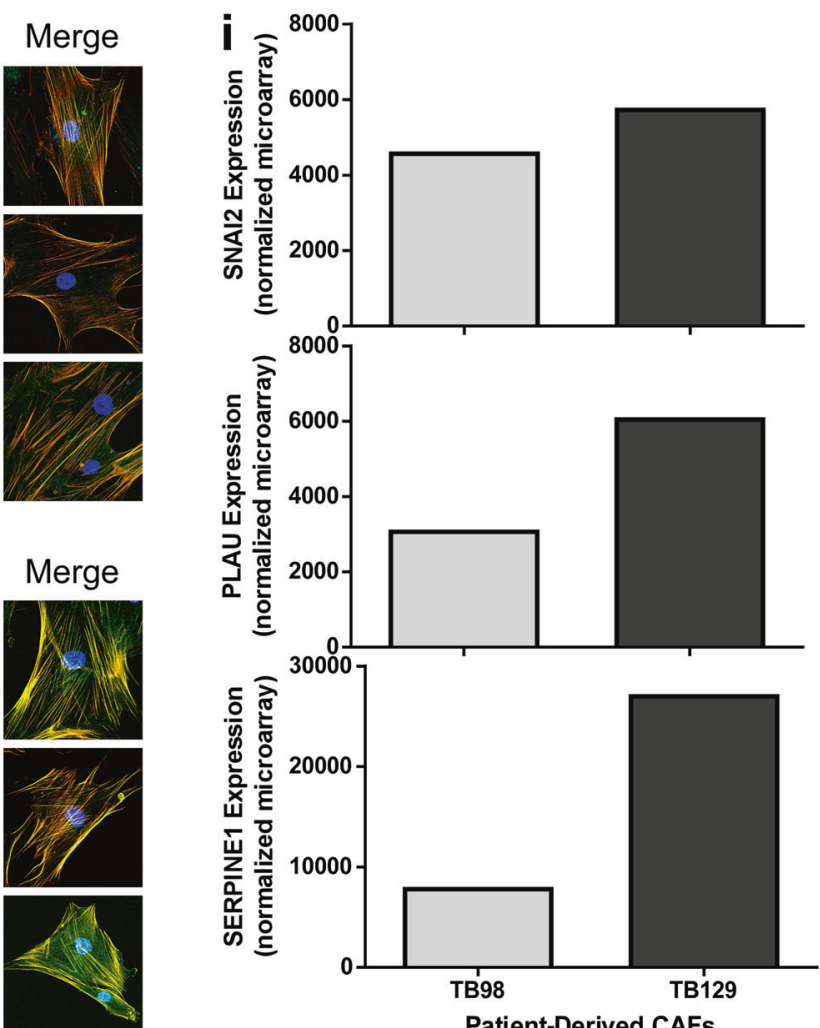

Fig. 2 SNAI2 and PEAK1 coexpression in breast cancers enriched for mesenchymal stem cell content is prognostically unfavorable. a-c. Kaplan-Meier OS curves for low or high PEAK1 transcript levels in breast cancer patients selected for high SNAI2 expression and enriched innate immune (a), adaptive immune (b) or mesenchymal stem cell contents ( $n=45,19$ and 382, respectively). d Kaplan-Meier OS curves for low or high PEAK1 transcript levels in breast cancer patients selected for high SNAI2 expression and decreased mesenchymal stem cell content $(n=381)$. e Western blot for PEAK1 and GAPDH in total lysates from the indicated non-tumorigenic cell lines. $\mathbf{f}$ Western blot and relative band intensity for PEAK1 and $\beta$-actin in total lysates from the indicated patient-derived breast cancer-associated fibroblasts. Representative confocal microscopy images for nucleus (DAPI), filamentous actin (Phalloidin) and PEAK1 in TB98 (g) and TB129 (h) CAF lines plated onto $5 \mathrm{ug} / \mathrm{mL}$ collagen, fibronectin or laminin substrates. i Normalized expression of SNAI2, PLAU and SERPINE1 in the indicated TB CAF lines obtained from the GEO database using the GSE37614 dataset.

reduced RFS (Fig. 1n, o) as well as DMFS (Supplementary Fig. 3a) in HER2-positive breast cancer - patterns also observed for fibronectin in this same cancer subset (Supplementary Fig. 3c). In support of a cooperative role for PEAK1 and SNAI2 within the same fibroblastic stromal cells in HER2-positive breast cancers, the poor prognostic utility of PEAK1 in HER2-positive breast cancer was notably restricted to patient tumors expressing high levels of SNAI2 (Fig. 1p-s and Supplementary Fig. 3). 


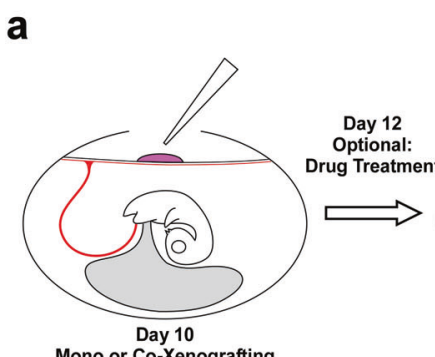

Mono or Co-Xenografting

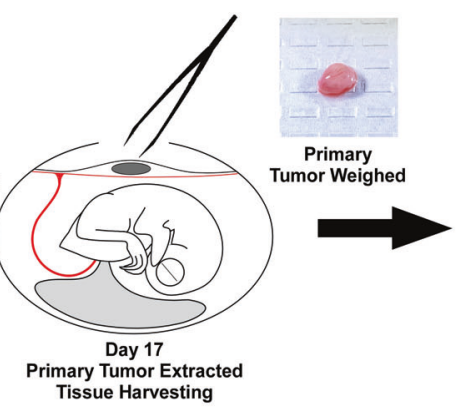

Tissue Harvesting

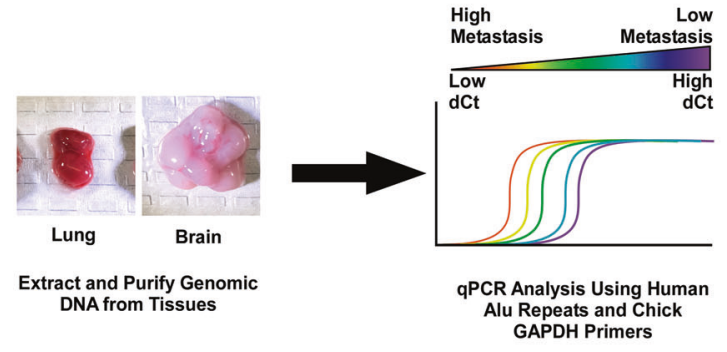

b

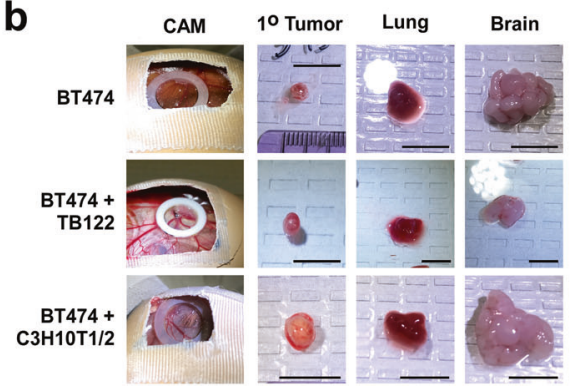

C

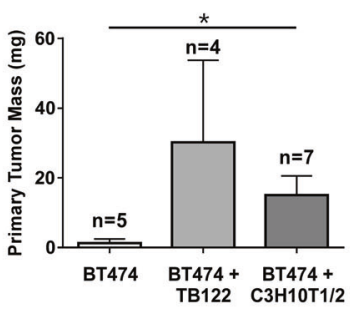

d

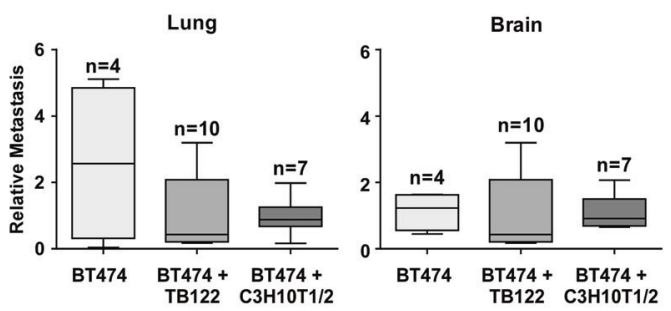

Fig. 3 Chicken embryo chorioallantoic membrane (CAM) xenografting of patient-derived CAFs or MSCs with HER2-positive breast cancer cells increases primary tumor mass. a Schematic of the chorioallantoic membrane (CAM) xenograft system using chicken (Gallus gallus) embryos together with human tumor cells and end point analysis of whole tissue genomic DNA by qPCR for human alu repeats and host chicken Gapdh levels. The method has been modified from the original assay system [32] to enable a 5-day drug treatment regimen beginning at 2 days post-xenograft. b Representative images of BT474 cells, BT474 cells + TB122 CAFs or BT474 cells + C3H10T1/2 mesenchymal stem cells. Scale bar $=1 \mathrm{~cm}$. c Quantified primary tumor mass of experiments represented in b. d Relative metastasis of BT474 cells in the lung (left) and brain (right) of experiment in b. *Indicates a $p$ value $<0.05$ as determined by a One-Way ANOVA w/ multiple comparisons post-test.

SNAI2 and PEAK1 coexpression in breast cancers enriched for mesenchymal stem cell content is prognostically unfavorable We next mined clinical data for relationships between high coexpression of SNAI2 and PEAK1 and OS across patient tissues enriched for specific stromal cell types. High expression of both SNAI2 and PEAK1 did not predict OS probability among breast cancer patients reporting enrichments in either innate or adaptive immune cell content (Fig. 2a, b). However, high expression of both SNAI2 and PEAK1 predicted significantly lower OS among patients with mesenchymal stem cell (MSC) content (Fig. 2c). In contrast, high PEAK1 expression levels among patients with high SNAI2 expression was not prognostically significant in patients with decreased MSC content (Fig. 2d).

By evaluating PEAK1 expression across non-tumor cell types (i.e., 3 fibroblast-like, 1 endothelial and 1 innate immune cell lines), we further established that PEAK1 expression was highest within fibroblast-like cell types (Fig. 2e). These data were further supported by analyzing the expression of PEAK1 across a subset of patient-derived cancer-associated fibroblasts (CAFs) (i.e., two from each breast cancer subtype) previously isolated and transcriptomically profiled [30] (Fig. 2f). At the subcellular level, PEAK1 localized strongly with the actin cytoskeleton in both the TB98 (ER-positive subtype) and TB129 (HER2-positive subtype) CAFs independent of extracellular matrix (ECM) substrate (Fig. $2 \mathrm{~g}$, $\mathrm{h}$ and Supplementary Fig. 4). Finally, analysis of SNAI2 and two other mesenchymal stromal cell genes (i.e., PLAU and SERPINE1) revealed their collective expression to be higher in the TB129 CAF line relative to the TB98 line (Fig. $2 \mathrm{i}$ ).

\section{Chicken embryo chorioallantoic membrane (CAM)} xenografting of patient-derived CAFs or MSCs with HER2positive breast cancer cells increases primary tumor mass We next asked whether PEAK1-expressing CAFs or MSCs could affect breast tumor growth and progression in the Gallus gallus embryo chorioallantoic membrane (CAM) in vivo model [31-33]
(Fig. 3a). As shown in Fig. 3b, c, the mass of BT474-derived tumors significantly greater when xenografted together with either the TB122 CAFs or C3H10T1/2 MSCs, although neither the CAF- nor MSC-containing xenografts displayed a measurable difference in early metastatic dissemination events to lung or brain tissues (Fig. 3d). Similarly, MCF7 cells xenografted together with TB130 CAFs or together with TB130 CAFs after in vitro pre-incubation with TB130 $\mathrm{CAF}$ conditioned media (CM) formed larger primary tumors while differences in early metastatic dissemination events to the lung and brain tissue were not observed (Supplementary Fig. 5). These results support using this system, in agreement with previous reports [34-36], to interrogate the role of PEAK1 in MSC-mediated HER2-positive breast cancer progression and targeted therapy response.

\section{Knockdown of PEAK1 in MSCs abrogates their ability to} promote tumorigenesis, intratumoral aSMA expression, lapatinib resistance and lapatinib-induced brain metastasis A panel of stable shRNA C3H10T1/2 MSC derivatives containing either a scramble control shRNA construct (shScr) or one of five PEAK1-targeting shRNAs (shP1) was generated (Fig. 4a). As before with the parental C3H10T1/2 MSCs, xenografting the shScr MSCs with the BT474 cells significantly increased primary tumor mass an effect that was abrogated by PEAK1 knockdown using two unique shRNA constructs (Fig. 4b). Notably, PEAK1-expressing MSCs caused BT474 tumors to contain elevated alpha-smooth muscle actin (aSMA) staining and vascular-like structures (Fig. 4c).

We then analyzed whether these MSCs could render BT474 tumors resistant to lapatinib treatment in vivo and whether any observed effects might require PEAK1 expression. Notably, shScr MSCs rendered BT474 cells less sensitive to lapatinib in vivo. Furthermore, BT474 xenografts containing MSCs with the PEAK1targeting shRNAs responded to lapatinib as though there were no MSCs xenografted (Figs. 4d, e). In agreement with these data, hematoxylin and eosin $(\mathrm{H} \& \mathrm{E})$ staining revealed a high degree of 
a

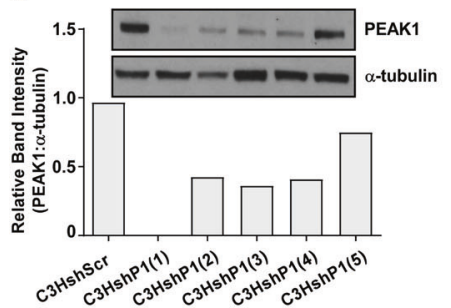

d $\mathrm{BT} 474$

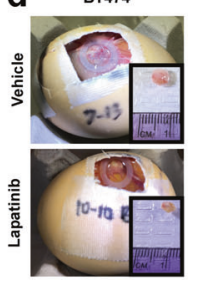

f
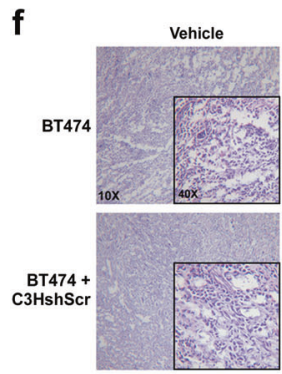

BT474 +
C3HshP1

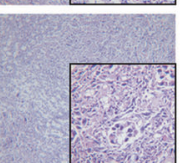

$\underset{\text { Lapatinib }}{300 \mathrm{nM}}$

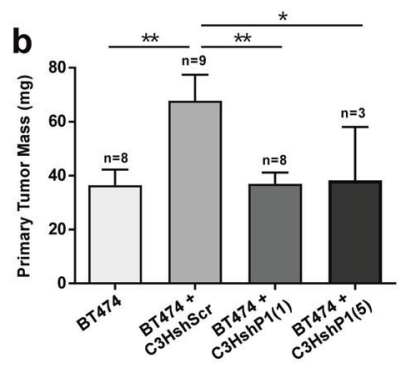

c

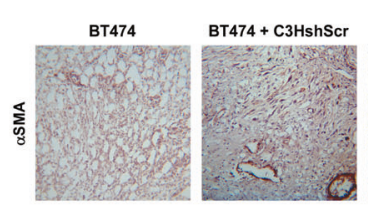

$\mathrm{BT} 474$ + $33 \mathrm{HshP} 1$

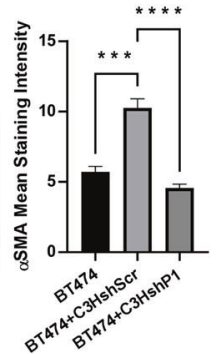

e
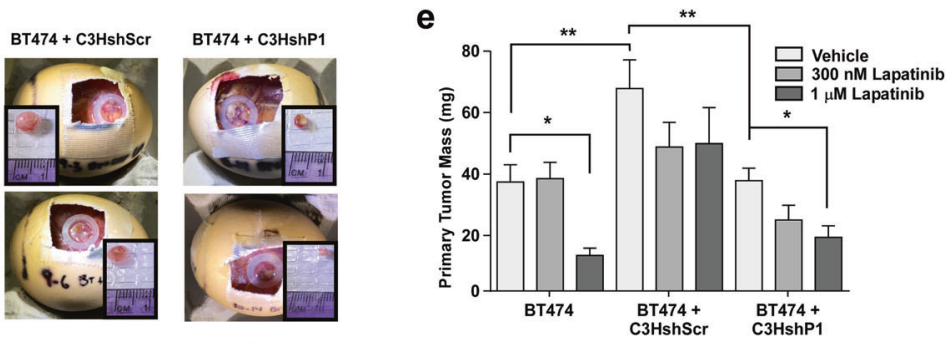

g
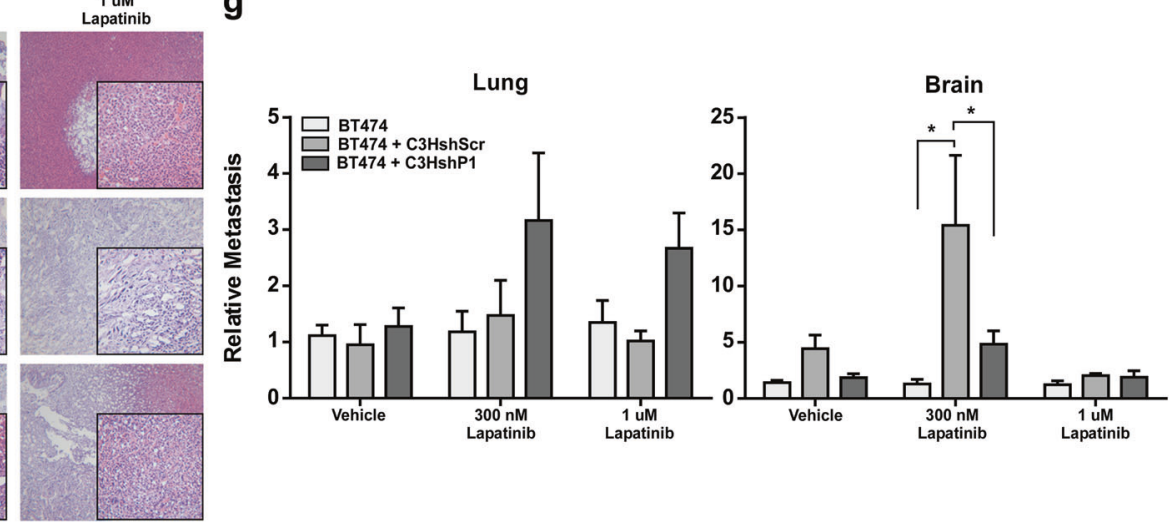

Fig. 4 Knockdown of PEAK1 in MSCs abrogates their ability to promote tumorigenesis, intratumoral aSMA expression, lapatinib resistance and lapatinib-induced brain metastasis. a Western blot and relative band intensity quantification for PEAK 1 and $\alpha$-tubulin levels in shScramble control (shScr) and 5 different PEAK1-targeting (shP1) shRNA derivatives of C3H10T1/2 mesenchymal stem cells. Unless otherwise noted, shP1(1) construct is used throughout experiments. b Quantified primary tumor mass of CAM xenograft assay using BT474 cells only or BT474 cells xenografted together with the $\mathrm{C} 3 \mathrm{H} s h \mathrm{Scr}, \mathrm{C} 3 \mathrm{HshP1}(1)$ or $\mathrm{C} 3 \mathrm{H} s h P 1(5)$ cells. c Representative images and quantification of alphasmooth muscle actin staining of stromal tissue in CAM tumors from $\mathbf{b}$. $\mathbf{d}$ Representative assay endpoint images for CAM xenograft experiment using the same cell combinations as in b with either vehicle control or 1 uM lapatinib treatments. e Quantified primary tumor mass of experiment in $\mathbf{d}$ including the treatment condition of $300 \mathrm{nM}$ lapatinib. $\mathbf{f}$ Hematoxylin and eosin staining of CAM tumor tissue in d. $\mathbf{g}$ Relative metastasis of BT474 cells in the lung (left) and brain (right) of experiment in d. ${ }^{*},{ }^{* *},{ }^{* *}$ and ${ }^{* * *}$ indicates a $p$ value $<0.05,0.01,0.001$ and 0.0001 , respectively, as determined by a One-Way or Two-Way ANOVA w/ multiple comparisons post-test.

necrotic tissue around the periphery of tumors generated from only BT474 cells alone or BT474 xenografts containing MSCs with the PEAK1-targeting shRNAs at both lapatinib doses (Fig. 4f). Interestingly, the presence of PEAK1-expressing MSCs in primary tumors treated with intermediate doses of lapatinib enabled the BT474 cells to escape and metastasize to the brain at a 15 -fold greater frequency when compared to xenografts of the BT474 cells alone or BT474 cells and ShP1 MSCs (Fig. 4g).

\section{MSC expression of PEAK1 protects neighboring breast cancer cells from lapatinib-induced cytotoxicity}

To elucidate potential mechanisms by which these stromal cells elicit their tumor- and lapatinib resistance-promoting functions, we employed co-culture methods [13] to further evaluate whether MSCs or breast fibroblasts could promote breast cancer cell expansion and resistance to lapatinib in vitro (Fig. 5a). Co-seeding either MSCs or AR22 breast fibroblasts together with H2B-eGFP labeled BT474 cells established monolayer co-cultures in which the breast cancer cells formed islands surrounded by fibroblasts (Fig. 5a and Supplementary Fig. 4a). We then used this system in combination with IncuCyte imaging to evaluate both the number of eGFP-positive and EtBr-positive breast cancer cells during timecourse lapatinib dose-response experiments (Fig. 5a and Supplementary Fig. 6a). As shown in Supplementary Fig. 6b, monocultures of either the MSCs or fibroblasts did not respond to increasing lapatinib doses as measured by EtBr uptake while the BT474 mono-cultures did, demonstrating that this targeted therapy displays specific cytotoxicity to HER2-overexpressing breast cancer cells. Interestingly, while co-culture of BT474 cells together with MSCs was able to both increase the basal number of BT474 cells (Fig. 5b) and reduce lapatinib cytotoxicity (Fig. 5c), the breast fibroblasts seemed to selectively reduce lapatinib cytotoxicity (Fig. 5c and Supplementary Fig. 6b). Using the shScr and ShP1 MSC derivatives in this co-culture assay revealed that PEAK1 expression mediates the ability for MSCs to protect neighboring breast cancer cells against lapatinib-induced cytotoxicity (Fig. $5 d$ ).

PEAK1 expression in MSCs drives the production of secreted factors that promote breast cancer cell proliferation/survival and lapatinib resistance in vitro

We also tested conditioned media (CM) from these cell types for their ability to promote breast cancer cell expansion and/or 
a
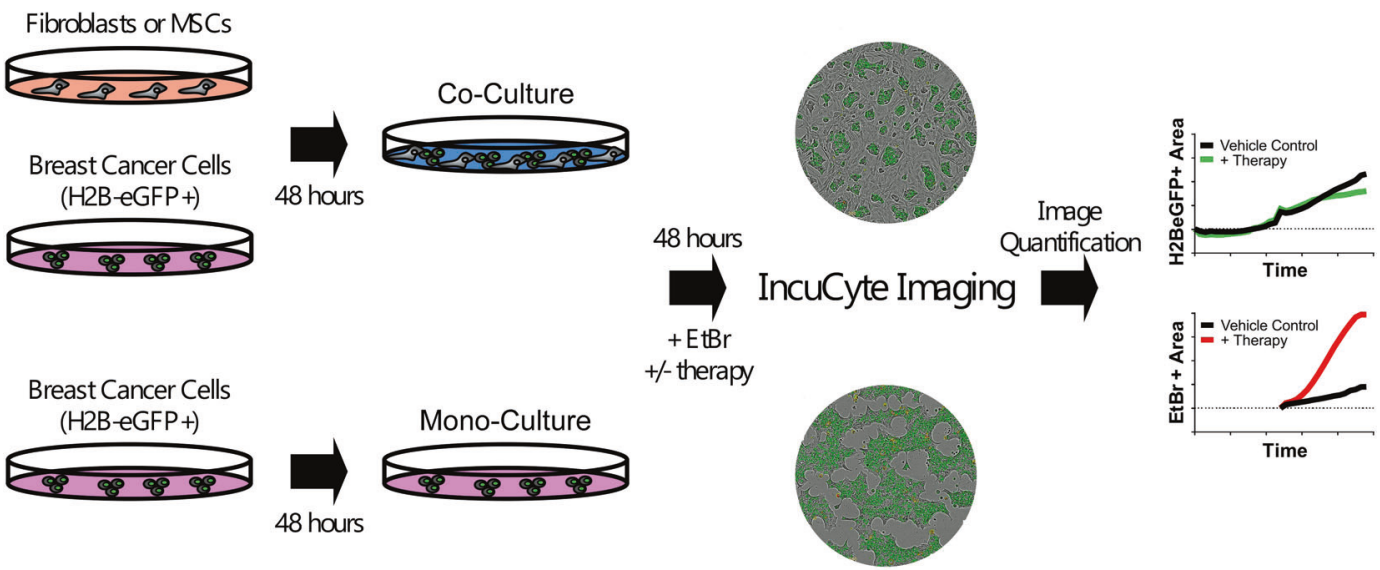

48 hours

C
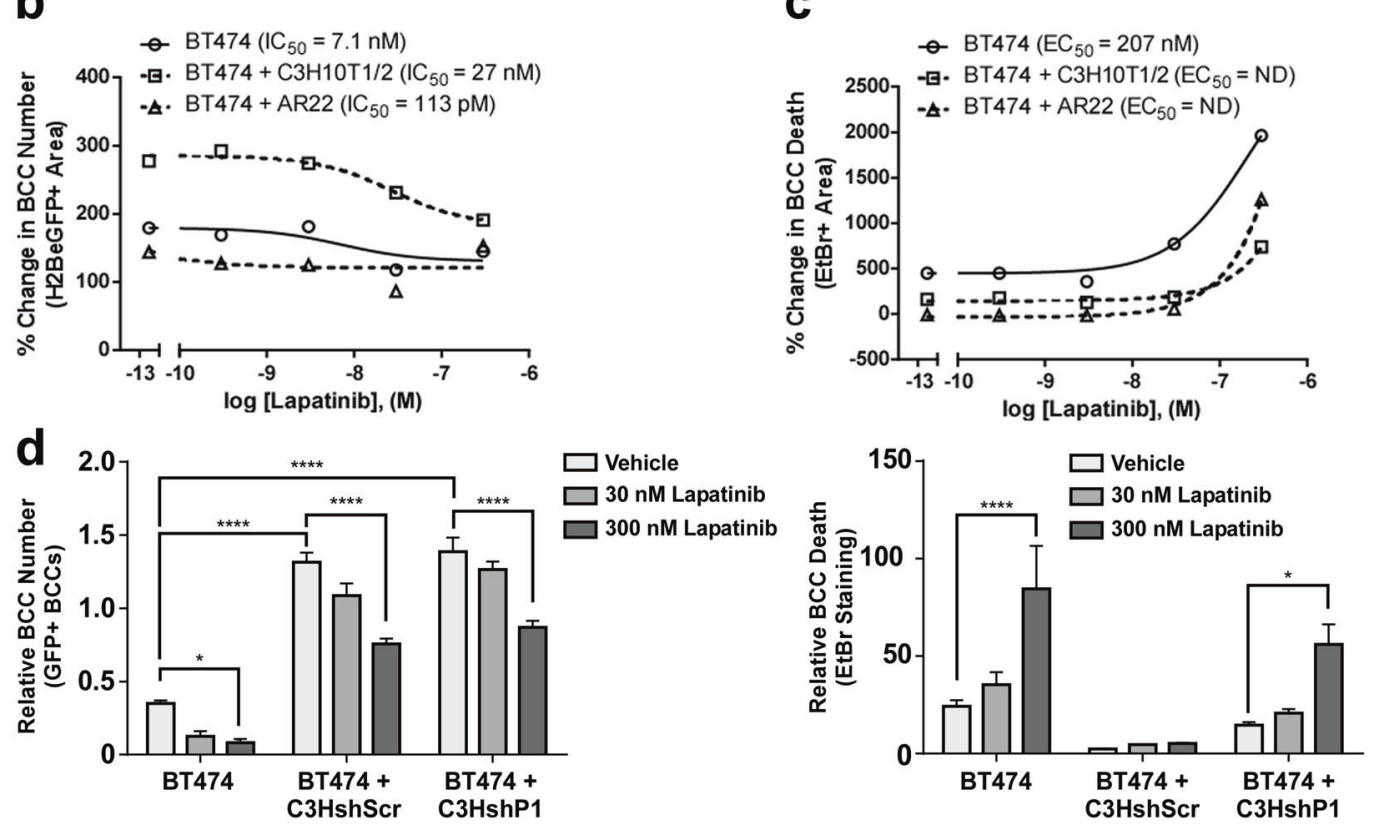

Fig. 5 MSC expression of PEAK1 protects neighboring breast cancer cells from lapatinib-induced cytotoxicity. a Schematic of breast cancer cell mono- or breast cancer cell-CAF/MSC co-culture using non-labeled stromal fibroblasts and H2B-eGFP+ BT474 breast cancer cells for downstream analysis of breast cancer cell number and death using the IncuCyte imaging system over $96 \mathrm{~h}$ ( $48 \mathrm{~h}$ pre-incubation and $48 \mathrm{~h}$ incubation with therapy and $\mathrm{EtBr})$. b-c Endpoint dose-response curves for lapatinib effects on breast cancer cell number (b) or cell death (c) in the indicated breast cancer cell and stromal fibroblast culture combinations. d Quantification of tumor cell number (left) and EtBr uptake (right) at assay endpoint for BT474 cells alone or co-cultured with the shScr or shP1(5) derivatives of C3H10T1/2 cells and treated with vehicle control or the indicated dose of lapatinib. ${ }^{*}$ of ${ }^{* * *}$ indicates a $p$ valule of 0.05 or 0.0001 , respectively as determined by a Two-Way ANOVA with multiple comparisons post-test.

lapatinib resistance in vitro (Fig. 6a). CM collected from CAFs derived from either HER2-positive (Fig. 6b) or ER-positive (Supplementary Fig. 7a) breast cancers potentiated BT474 or MCF7 cell growth, respectively. Notably, by using shRNA MSC derivatives, PEAK1 was found to be necessary to produce secreted factors into MSC CM that potentiate BT474 (Fig. 6c) or mouse Py230 cell growth (Supplementary Fig. 7b). Finally, MSC expression of PEAK1 was necessary for MSC-derived CM to promote BT474 cell resistance to lapatinib (Fig. 6d).

PEAK1-dependent INHBA/activin-A expression/secretion from MSCs mediates MSC-induced lapatinib resistance in HER2positive breast cancer cells

To identify the factors that PEAK1 regulates within MSCs, we performed semi-quantitative protein array analysis targeting 308 protein antigens in lysates from the shScr and two unique shP1
MSC derivatives (Fig. 7a). PEAK1 knockdown led to a greater than 2-fold decrease in 5 proteins (GDF5, CCR4, INHBA/activin-A, GRH and $(C L 4)$ and a greater than 1.8-fold increase in 7 additional proteins (PDGFRA, CSF1, HGFR, Frizzled-6, VEGFA, PF4 and TGFB3) (Dataset 1). As shown in Fig. 7b, six PEAK1-dependent soluble factors met the $95 \%$ confidence interval cut-off criteria for further analysis. We next sought to determine whether there was any clinical relevance of these PEAK1-regulated MSC gene/proteins (i.e., TGFB3, VEGFA, CSF1, CCL4, INHBA and GDF5). As with PEAK1 (Fig. 1), we analyzed expression profiles for these genes across two independent studies investigating normal breast stroma and malignant breast stroma $[6,29]$. Notably, the transcripts of PEAK1suppressed MSC factors (i.e., TGFB3, VEGFA and CSF1) were significantly lower within breast cancer stroma (Fig. 7c, left three graphs). In contrast, the factors whose expression was dependent upon PEAK1 in MSCs (i.e., CCL4, INHBA and GDF5) displayed 
a

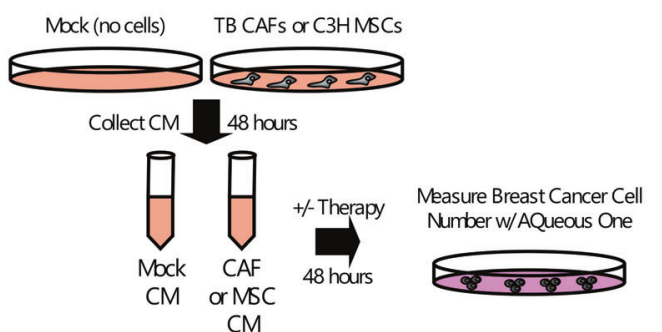

C

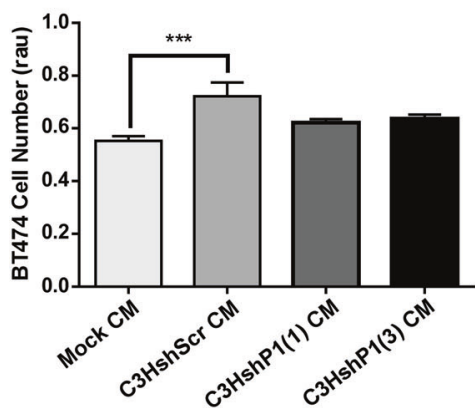

b
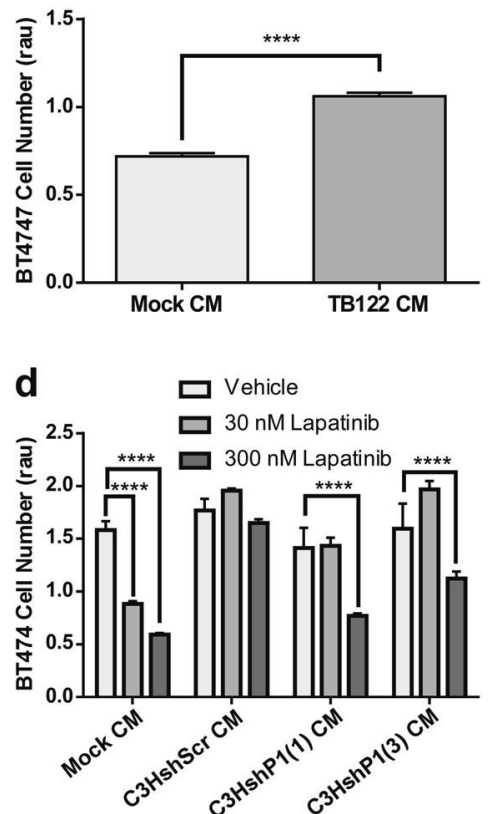

Fig. 6 PEAK1 expression in MSCs drives the production of secreted factors that promote breast cancer cell proliferation/survival and lapatinib resistance in vitro. a Schematic for generating TB CAF or C3H MSC conditioned media (CM) for analysis on breast cancer cell growth/survival over $48 \mathrm{~h}$ in vitro. b Cell viability analysis of BT474 cells treated with mock or TB122 CM. c Cell viability analysis of BT474 cells treated with mock CM or CM from the indicated shRNA derivatives of C3H10T1/2 cells. $\mathbf{d}$ Cell viability analysis of BT474 cells treated with mock CM or CM from the indicated shRNA derivatives of $\mathrm{C} 3 \mathrm{H} 10 \mathrm{~T} 1 / 2$ cells and treated with vehicle control or the indicated dose of lapatinib. *, ***, or **** indicates a $p$ value $<0.05,0.001$ or 0.0001 , respectively, as determined by a One-Way or Two-Way ANOVA w/multiple comparisons posttest.

significantly higher transcript levels in breast cancer stroma across both studies, with INHBA/activin-A showing the greatest average fold-change increase in malignant over normal breast stroma (Fig. 7c, right three graphs). Analysis of the mRNA expression relationship between PEAK1 and each of these six genes in breast cancer patient tissues [37] revealed that PEAK1 and INHBA transcripts showed the most significant positive correlation (Fig. 7d). Additional analysis of RFS and OS based upon elevated expression for each of these six factors in HER2-positive together with PEAK1 or an enriched MSC signature (Supplementary Fig. 8a-c) highlighted the prognostic importance for INHBA/activin-A. ELISA analysis was performed on mock media or conditioned medias from the shRNA MSC derivatives and revealed that soluble activinA was only detectable in the conditioned media from PEAK1expressing $\mathrm{C} 3 \mathrm{H}$ MSCs (Fig. 7e). Finally, using two unique activin-A antagonists (i.e., Follistatin and ACTRII-ECD), we demonstrate that the lapatinib protective effects of MSC conditioned media requires activin-A (Fig. 7f, g).

PEAK1-expressing MSCs promote lapitinib resistance by modulating antiapopototic/DNA damage signaling within a subpopulation of highly plastic HER2-positive breast cancer cells

To capture HER2-positive tumor cell states changes in the presence of PEAK1-expressing MSCs and in response to lapatinib treatment, we performed single-cell Cyclic Immunofluorescence (CyclF) as previously described [26] in combination with the coculture system described in Fig. 5a (Fig. 8a) across seven unique cell state markers measuring apoptosis evasion (i.e., MCL1), growth signaling (i.e., p-Akt), metastasis (i.e., VIM), DNA damage (i.e., $\mathrm{p}-\mathrm{\gamma H} 2 \mathrm{AX}$ ), oxidative response (i.e., p65NFkB), stress response (i.e., GRP78) and microenvironment fibroblast activation (i.e., aSMA). Single-cell quantification of these antigen markers across three biological replicates for four cell culture conditions and three lapatinib treatment conditions produced $3,462,844$ data points in 494,692 single cell events. T-distributed stochastic neighbor embedding (t-SNE) was used to reduce data dimensionality (Fig. $8 \mathrm{~b}$ ) and to gate on GFP-positive breast cancer cells (Fig. 8c). This analysis demonstrated good cell resolution and lapatinib-induced reduction in breast cancer cell number that was significantly blocked by the presence of PEAK1-expressing MSCs (Fig. 8d, e). Inspection of the t-SNE outputs for BT474 cell monocultures or the BT474 + C3HshRNA cocultures across control, $30 \mathrm{nM}$ or $300 \mathrm{nM}$ lapatinib treatment conditions (Supplementary Fig. 9a) allowed us to identify four breast cancer cell and five MSC subpopulations that emerged in $\mathrm{BT} 474+\mathrm{C} 3 \mathrm{H}$ shScr cocultures and persisted in the presence of lapatinib (Fig. 8f). p-Akt, p65NFkB, p- $\gamma \mathrm{H} 2 \mathrm{AX}, \mathrm{MCL} 1$, GRP78 and VIM markers were expressed highest in the GFPpositive breast cancers while aSMA was expressed predominantly in the GFP-negative MSCs (Fig. 8g). In agreement with our in vivo data (Fig. 4c), we observed a striking PEAK1-dependent increase in aSMA expression across all populations of MSCs in co-culture with BT474 cells (Supplementary Fig. 9b).

Systematic analysis of the antigen integrated signal densities in GFP-positive breast cancer cells initially gated for positive/ negative expression of each of the other antigen markers (Supplementary Fig. 9c-f) revealed three unique subpopulations of breast cancer cells enriched in the presence of MSCs (i.e., p65NFKB ${ }^{\text {high }} / \mathrm{VIM}^{\text {high }}, \mathrm{p}-\mathrm{Akt}^{\text {high }} / \mathrm{VIM}^{\text {high }}$ and MCL $1^{\text {high }} / \mathrm{VIM}^{\text {high }}$ ) that were not dependent upon MSC expression of PEAK1 (Fig. 8h-j) and three unique subpopulations enriched in the presence of only PEAK1-expressing MSCs (i.e., p-Akt ${ }^{\text {high }} / \mathrm{p}-\mathrm{\gamma H}_{2} \mathrm{AX}{ }^{\text {low }}, \mathrm{MCL} 1^{\text {high }} / \mathrm{p}$ $\mathrm{pH}_{2} \mathrm{AX}^{\text {low }}$ and GRP78 ${ }^{\text {high }} / \mathrm{VIM}^{\text {high }}$ ) (Fig. $8 \mathrm{k}-\mathrm{m}$ ). Notably, these $\mathrm{p}$ $\mathrm{Akt}^{\text {high }} / \mathrm{p}-\mathrm{\gamma H}_{2} \mathrm{AX} \mathrm{X}^{\text {low }}, \mathrm{MCL}^{\text {high }} / \mathrm{p}-\mathrm{\gamma H} 2 \mathrm{AX}{ }^{\text {low }}$ and GRP78 ${ }^{\text {high }} / \mathrm{NIM}^{\text {high }}$ subpopulations persisted in the presence of lapatinib treatment (Fig. $8 n-p)$. In agreement with these data, immunofluorescence for 

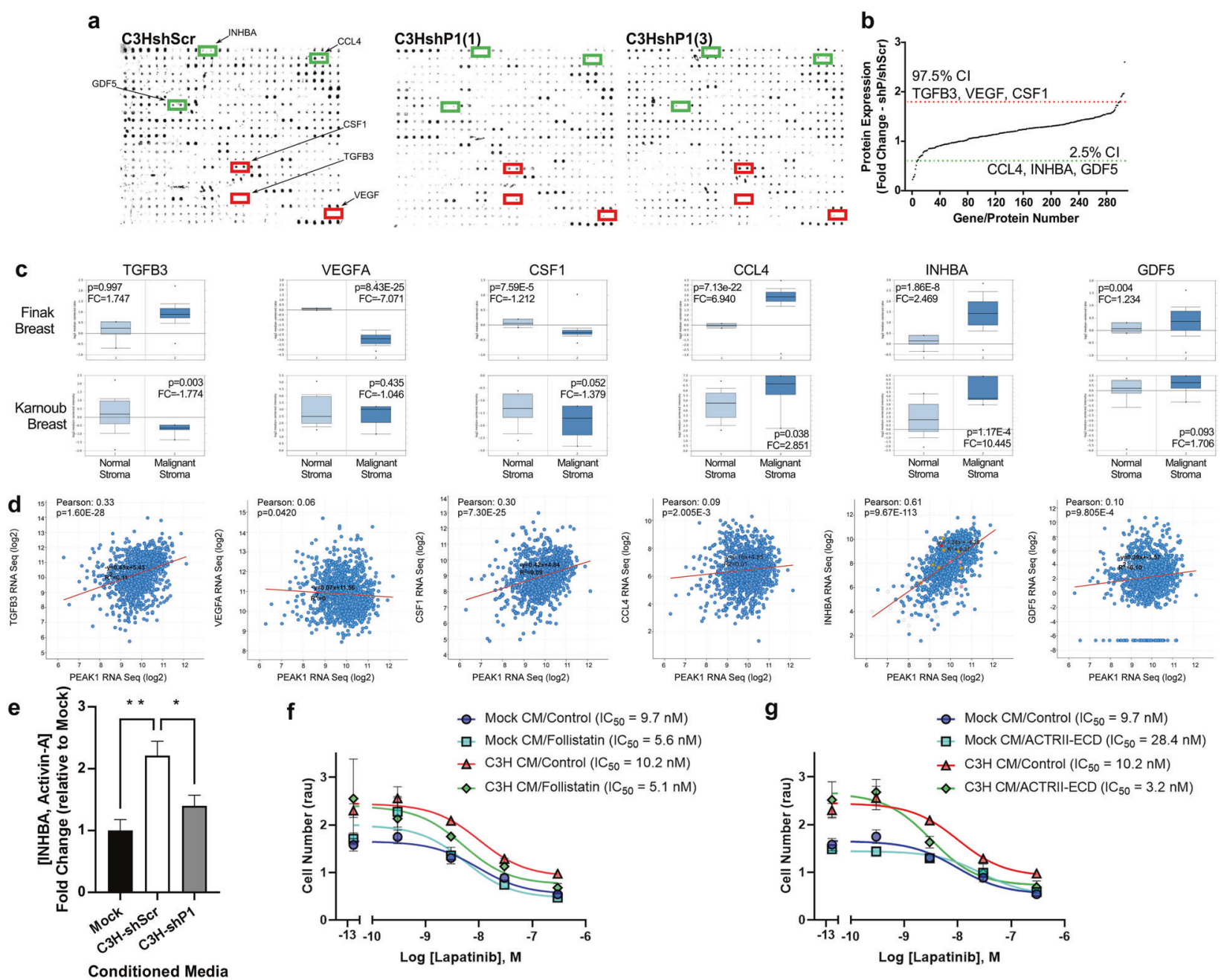

Fig. 7 PEAK1-dependent INHBA/activin-A expression/secretion from MSCs mediates MSC-induced lapatinib resistance in HER2-positive breast cancer cells. a Representative slide scan images from the semi-quantitative mouse antibody array 308 (L-308) following incubation and reactivity with total cell lysates prepared from the shScramble and indicated PEAK1-specific shRNA derivatives of the C3H10T1/2 MSCs. b Quantification of ranked protein expression across the 308 array antigens with confidence intervals set to identify antigen expression changes up (red line) and down (green) with a $p$ value $<0.05$. c Relative mRNA expression for the indicated PEAK1-dependent cytokines in normal versus malignant stroma as reported in the indicated studies. d Expression relationship for TGFB3, VEGFA, CSF1, CCL4, INHBA and GDF5 versus PEAK1 mRNA levels in breast cancer patients. e ELISA analysis for activin-A in mock conditioned media or media conditioned with the indicated shRNA derivatives of the C3H10T1/2 cells. f-g Aqueous One cell viability assay on BT474 cells treated with either mock or $\mathrm{C} 3 \mathrm{H} 10 \mathrm{~T} 1 / 2$ cell conditioned media and increasing doses of lapatinib in the presence of control or $1 \mu \mathrm{g} / \mathrm{mL}$ Follistatin (f) or ACTRII-ECD (g).

MCL1 and $\mathrm{p}-\mathrm{\gamma H} 2 \mathrm{AX}$ across the four cell culture conditions demonstrate that co-culture of BT474 cells with PEAK1expressing MSCs encircled $M C L 11^{\text {high }} / \mathrm{p}-\mathrm{gH} 2 \mathrm{AX}{ }^{\text {low }}$ cells in the presence of lapatinib (Fig. 8q)

Finally, we used Ingenuity Pathway Analysis (IPA) to evaluate the pathway, disease and functional annotations for a 10 gene set comprised of PEAK1, PEAK1-associated/dependent genes from the mesenchymal tumor stroma (i.e., SNAI2, INHBA, CCL4 and GDF5) and markers identified in our CyclF screen to be enriched in HER2-positive breast cancer cells when exposed to PEAK1-expressing MSC (i.e., AKT1, H2AFX, MCL1, GRP78 and VIM) (Fig. 8r-s). Expanded interactome analysis of these core genes using Cytoscape, generated 9 subnetworks that included enriched gene ontologies for regulation of transcription, antiapoptosis, mesoderm morphogenesis, stress responses, DNA damage responses, mesenchymal cell survival, exctracellular matrix (ECM) disassembly and JAK-STAT signaling (Supplementary Fig. 10). Taken together, we identify a pharmacologically targetable PEAK1-INHBA-dependent and SNAI2-associated stromal cell non-autonomous mechanism through which neighboring HER2-positive breast cancer cells increase mitogenic, antiapoptotic and stress signaling activity; acquire lapatinib resistance; and metastasize to the brain (Fig. 8t).

\section{DISCUSSION}

It is well-documented that targeted therapy resistance of HER2positive breast cancer strongly associates with the onset of brain metastasis [38]. Recent work has also reported that stromal fibroblast reprogramming by SNAI2 drives solid tumor progression and upregulated PEAK1 expression [39]. In this regard, we demonstrate that high PEAK1 expression in HER2-positive breast cancer patient tissues predicts increased disease relapse (Fig. 1b) in HER2-positive breast cancers high in SNAI2 expression (Fig. 1s and Supplementary Fig. 3a) and mesenchymal stem cell (MSC) content (Fig. 2c). Notably, we also demonstrate that HER2-positive breast cancers contain fibroblastic stromal cells positive for both cytoplasmic PEAK1 and nuclear SNAI2 (Fig. 1l, m). 
a

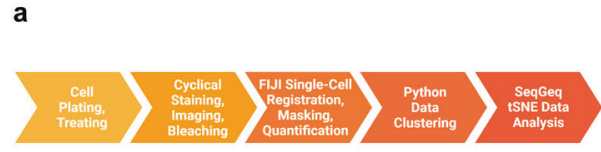

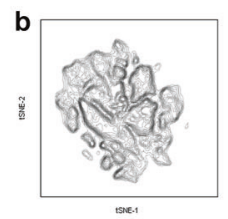
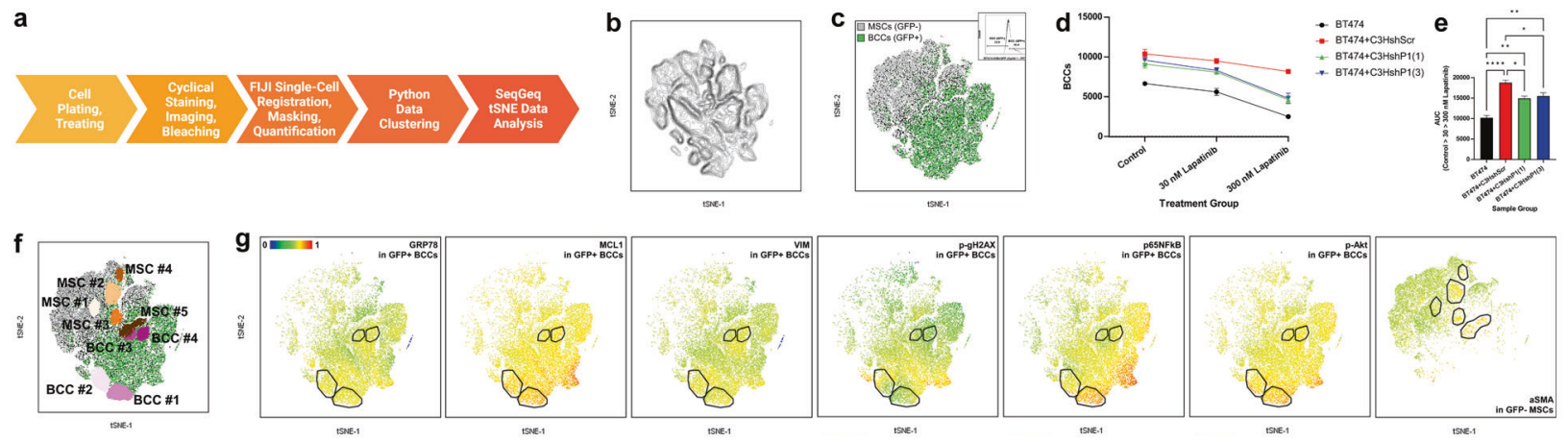

h
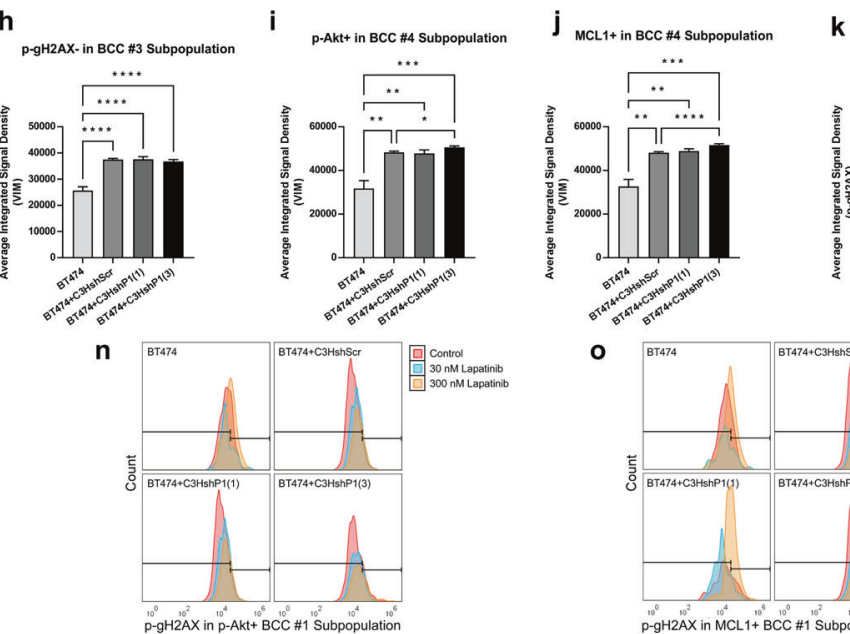

$q$
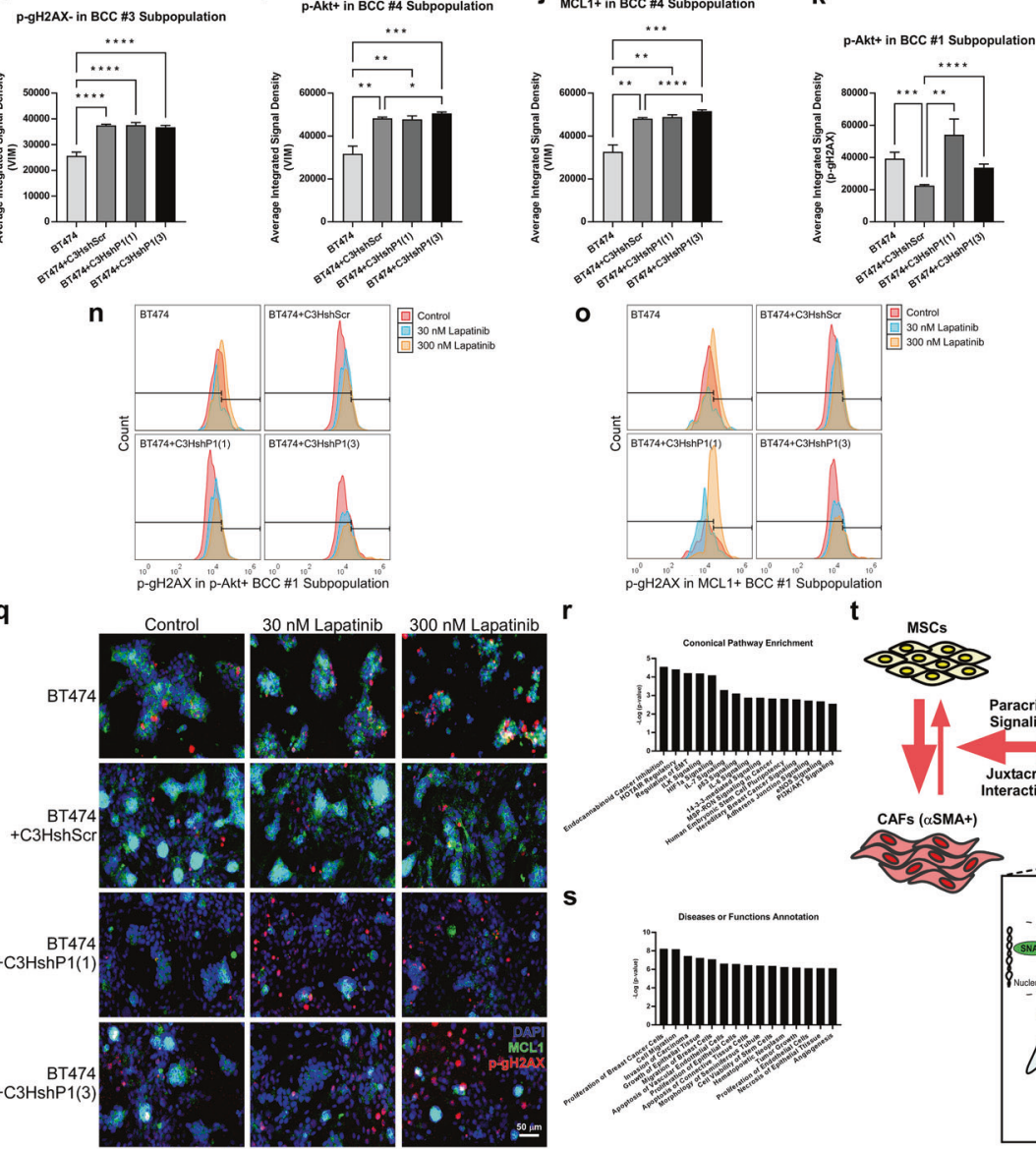

0
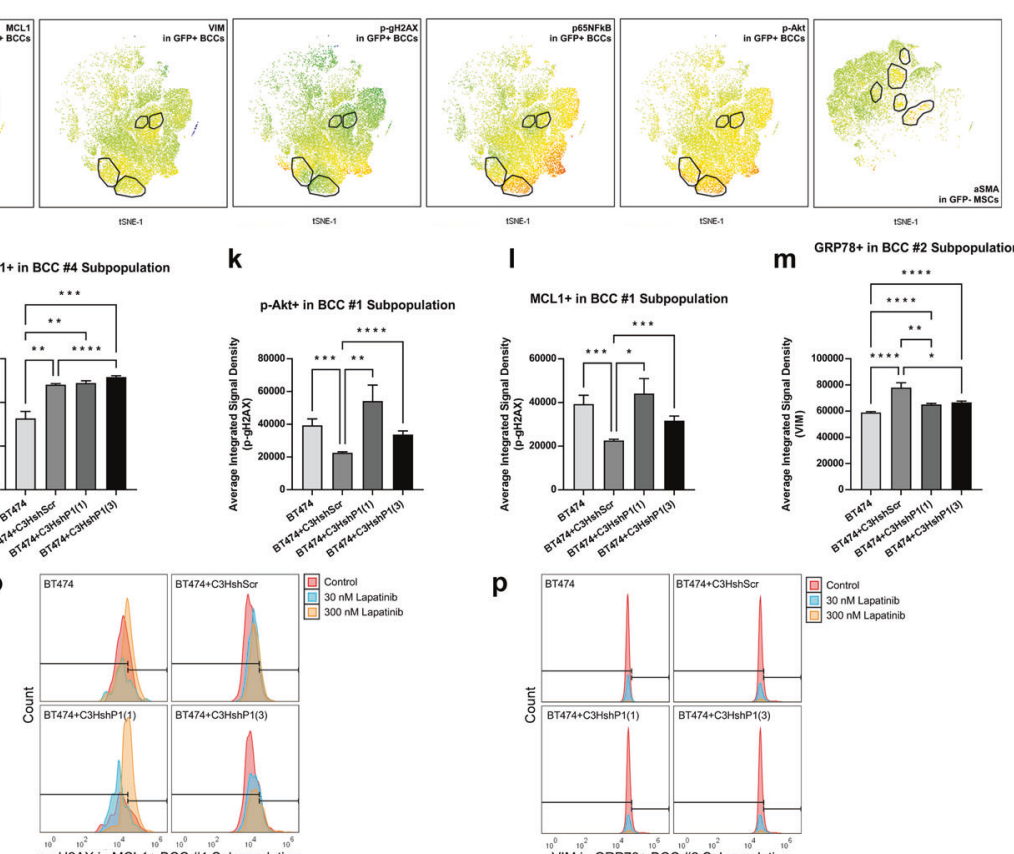

I

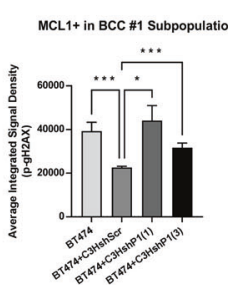

m

GRP78+ in BCC \#2 Subpopulation

p

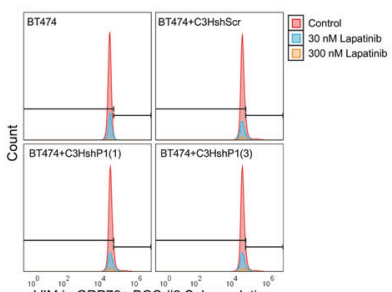

$t$ MSCs
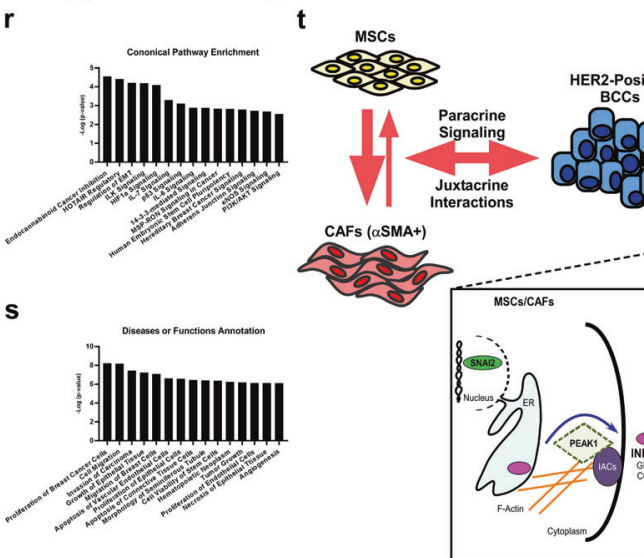

Fig. 8 PEAK1-expressing MSCs promote lapitinib resistance by modulating antiapopototic/DNA damage signaling within a subpopulation of highly plastic HER2-positive breast cancer cells. a Schematic of the CyclF workflow used for single-cell analysis. b-c tSNE plots of all cells across biological replicates of cell culture and lapatinib treatment conditions (b) and overlayed with the GFP-positive BT474 HER2-positive breast cancer cells (c, inlay shows GFP gating scheme). d Averaged BT474 GFP-positive breast cancer cell (BCC) number at $48 \mathrm{~h}$ post-therapy treatment. e Quantification of ar---ea under the curve (AUC) for data plotted in d. $\mathbf{f}$ Overlay of notable breast cancer cell and MSC subpopulations onto the t-SNE plot from c. g Pseudocoloring of the single-cell antigen intensities of GRP78, MCL1, VIM, p- $\gamma \mathrm{H} 2 \mathrm{AX}$ p65NFkB and p-Akt in the GFP-positive breast cancer cells and $\alpha$ SMA in the GFP-negative MSCs overlayed onto the t-SNE plots for these cell populations. h-m Average integrated signal intensity for indicated tumor cell markers within indicated gated populations of complementary markers across the four cell culture conditions. $\mathbf{n}-\mathbf{p}$ Histograms representing lapatinib-induced changes of the single-cell antigen expression patterns for $\mathrm{p}-\mathrm{Akt} \mathrm{high}^{\text {high }} / \mathrm{gH} 2 \mathrm{AX} \mathrm{X}^{\text {low }}, \mathrm{MCL}^{\text {high }} / \mathrm{p}-\mathrm{gH} 2 \mathrm{AX} \mathrm{X}^{\text {low }}$ and GRP78 ${ }^{\text {high }} / \mathrm{VIM}^{\text {high }} \mathrm{BT} 474$ cell subpopulations identified in $\mathbf{k}-\mathbf{m}$. q Representative microscopy images of nuclear (DAPI), MCL1 and $\mathrm{p}-\mathrm{gH} 2 \mathrm{AX}$ immunofluorescence across cell culture and lapatinib treatment conditions. IPAderived canonical pathway (r) or disease/function annotation enrichments (s) for SNAI2, PEAK1, INHBA, CCL4, GDF5, MCL1, AKT1, H2AFX, GRP78 and VIM. $\mathbf{t}$ Proposed model of mechanism by which stromal expression of PEAK1 drives tumor growth, metastasis and targeted therapy resistance in neighboring HER2-positive breast cancer cells.

Marusyk and colleagues previously demonstrated that coculturing or xenografting stromal fibroblasts together with HER2-positive breast cancer cells sustains Akt phosphorylation in the presence of lapatinib treatment [5]. More recently, Zervantonakis et al. reported that fibroblast-tumor cell signaling limits lapatinib treatment via the secretion of soluble factors which activate MTOR and antiapoptotic pathways across bulk tumor cells [13]. These results are also consistent with previous reports that MCL1 confers protection of HER2-positive breast cancer to environmental stress [40]. In this regard, we identify a new 
PEAK1-INHBA/activin-A-dependent axis in MSCs that is necessary for MSC-induced lapatinib resistance in HER2-positive breast cancer cells (Fig. 7). We further determine that PEAK1-expressing MSCs promote the emergence of $\mathrm{p}-\mathrm{Akt}^{\text {high }} / \mathrm{p}-\mathrm{\gamma H} 2 \mathrm{AX}{ }^{\mathrm{low}}, \mathrm{MCL} 1^{\text {high }}$ / $\mathrm{p}-\mathrm{\gamma H}_{2} \mathrm{AX}^{\text {low }}$ and GRP78 high $/ \mathrm{VIM}^{\text {high }}$ subpopulations within HER2positive breast cancer cells that display resistance to lapatinib and are capable of enhancing tumorigenesis in vitro and in vivo (Figs. 4-6 and 8). Thus, it will be instructive to test combinatorial inhibition of INHBA/activin-A, PI3KCA/AKT1, MCL1 and GRP78 (BiP) signaling as a means to overcome HER2-targeted therapy responses.

A role for MSCs in the breast cancer microenvironment as effectors of tumor growth and metastasis has been previously established [6, 41, 42]. Notably, we observed that MSC expression of PEAK1 was required for MSCs to induce metastatic spread of HER2-positive breast cancer cells to the brain in animals treated with lapatinib (Fig. 4g). We also noted that the primary tumors in these animals showed elevated stromal aSMA expression (Fig. 4c) and no appearance of lapatinib-induced cytotoxicity (Fig. 4f). It is notable, however, that MSCs did not function to promote breast cancer metastasis (Fig. 3d and Supplementary Fig. 3c) in our analyses. This is likely due to the sensitivity of the in vivo CAM tumor model and its ability to detect the earliest stages of tumor progression [32] - stages at which the previously described prometastatic effects of MSCs may not have been observable. While the specific cellular processes and/or molecular machinery governing these effects will require further characterization, one possibility is that stromal expression of PEAK1 enhances tumor vascularization. This is consistent with both our observation that HER2-positive breast cancer cells xenografted with MSCs displayed increased expression of aSMA and vascular architecture in a PEAK1-dependent manner (Fig. 4c) and recent work reporting a role for PEAK1 during developmental angiogenesis [43].

Previous analyses of cell line xenografts in mice and patient tumor tissue revealed that lapatinib treatment leads to a decreased distance between aSMA-positive stromal fibroblasts and proliferating HER2-positive breast cancer cells [5], implicating juxtacrine and/or distance-dependent paracrine signaling mechanisms such as those used by morphogens. We observed that PEAK1-dependent MSC-induced protection of HER2-positive breast cancer cells against lapatinib could occur in vivo (Fig. 4) and in vitro (Figs. 5 and 6). The possibility that these MSC-driven cytoprotective effects require one or more secreted factors is supported by our identification of six secreted/soluble proteins (i.e., TGFB3, VEGFA, CSF1, CCL4, INHBA and GDF5) that were expressed by MSCs in a PEAK1-dependent manner (Fig. 7a, b). While the mechanisms by which PEAK1 regulates the expression/ secretion of these factors remain to be determined, our data demonstrating that these MSC cytoprotective effects can be reversed by antagonism of activin-A in the MSC conditioned media (Fig. 7f, g) suggest that activin-A inhibition in HER2-positive breast cancers is a viable means for overcoming targeted therapy resistance. It is also notable that activins have well-established morphogen roles during normal development [44], and that previous work has reported increased INHBA/activin-A activity at the leading edge of HER2-positive breast tumors [45] and that follistatin (Fig. 7f) can suppress HER2-positive breast cancer metastasis [46]. These results together with our findings that PEAK1 expression predicts low median overall survival in breast cancer patients with high INHBA transcript levels and enriched for MSC content (Supplementary Fig. 8c), further support a role for PEAK1-dependent INHBA/activin-A expression as a mechanism by which stromal MSCs support HER2-positive breast cancer progression and therapy resistance. These studies establish a critical PEAK1-INHBA/activin-A stromal cell axis as a regulatory node that works in concert with SNAI2 to promote therapy resistance, metastasis and poor patient outcomes.

\section{REFERENCES}

1. Yuan S, Norgard RJ, Stanger BZ. Cellular plasticity in cancer. Cancer Discov. 2019;9:837-851.

2. Gupta PB, Pastushenko I, Skibinski A, Blanpain C, Kuperwasser C. Phenotypic plasticity: driver of cancer initiation, progression, and therapy resistance. Cell Stem Cell. 2019;24:65-78.

3. Runa F, Hamalian S, Meade K, Shisgal P, Gray PC, Kelber JA. Tumor microenvironment heterogeneity: challenges and opportunities. Curr Mol Biol Rep. 2017;3:218-229.

4. Marusyk A, Janiszewska M, Polyak K. Intratumor heterogeneity: the rosetta stone of therapy resistance. Cancer Cell. 2020;37:471-484.

5. Marusyk A, Tabassum DP, Janiszewska M, Place AE, Trinh A, Rozhok Al, et al. Spatial proximity to fibroblasts impacts molecular features and therapeutic sensitivity of breast cancer cells influencing clinical outcomes. Cancer Res. 2016;76:6495-6506.

6. Karnoub AE, Dash AB, Vo AP, Sullivan A, Brooks MW, Bell GW, et al. Mesenchymal stem cells within tumour stroma promote breast cancer metastasis. Nature. 2007;449:557-563.

7. Qu Y, Dou B, Tan H, Feng Y, Wang N, Wang D. Tumor microenvironment-driven non-cell-autonomous resistance to antineoplastic treatment. Mol Cancer. 2019;18:69.

8. Zoeller JJ, Bronson RT, Selfors LM, Mills GB, Brugge JS. Niche-localized tumor cells are protected from HER2-targeted therapy via upregulation of an anti-apoptotic program in vivo. NPJ Breast Cancer. 2017;3:18.

9. Cancer Genome Atlas N. Comprehensive molecular portraits of human breast tumours. Nature. 2012;490:61-70.

10. Arteaga CL, Sliwkowski MX, Osborne CK, Perez EA, Puglisi F, Gianni L. Treatment of HER2-positive breast cancer: current status and future perspectives. Nat Rev Clin Oncol. 2011;9:16-32.

11. Baselga J, Swain SM. Novel anticancer targets: revisiting ERBB2 and discovering ERBB3. Nat Rev Cancer. 2009;9:463-475.

12. Gori S, Montemurro F, Spazzapan S, Metro G, Foglietta J, Bisagni G, et al. Retreatment with trastuzumab-based therapy after disease progression following lapatinib in HER2-positive metastatic breast cancer. Ann Oncol. 2012;23:1436-1441.

13. Zervantonakis IK, Poskus MD, Scott AL, Selfors LM, Lin JR, Dillon DA, et al. Fibroblasttumor cell signaling limits HER2 kinase therapy response via activation of MTOR and antiapoptotic pathways. Proc Natl Acad Sci USA. 2020;117:16500-16508.

14. Wang Y, Kelber JA, Tran Cao HS, Cantin GT, Lin R, Wang W, et al. Pseudopodiumenriched atypical kinase 1 regulates the cytoskeleton and cancer progression [corrected]. Proc Natl Acad Sci USA. 2010;107:10920-10925.

15. Guth $R$, Adamian $Y$, Geller $C$, Molnar J, Maddela J, Kutscher $L$, et al. DHPSdependent hypusination of elF $5 \mathrm{~A} 1 / 2$ is necessary for TGFbeta/fibronectininduced breast cancer metastasis and associates with prognostically unfavorable genomic alterations in TP53. Biochem Biophys Res Commun. 2019;519:838-845.

16. Agajanian $M$, Runa $F$, Kelber JA. Identification of a PEAK1/ZEB1 signaling axis during TGF $\beta /$ fibronectin-induced EMT in breast cancer. Biochem Biophys Res Commun. 2015;465:606-612.

17. Agajanian M, Campeau A, Hoover M, Hou A, Brambilla D, Kim SL, et al. PEAK1 acts as a molecular switch to regulate context-dependent TGFbeta responses in breast cancer. PLoS One. 2015;10:e0135748.

18. Kelber JA, Reno T, Kaushal S, Metildi C, Wright T, Stoletov K, et al. KRas induces a Src/PEAK1/ErbB2 kinase amplification loop that drives metastatic growth and therapy resistance in pancreatic cancer. Cancer Res. 2012;72:2554-2564.

19. Ding $C$, Tang W, Fan X, Wang $X, W u ~ H, X u ~ H$, et al. Overexpression of PEAK1 contributes to epithelial-mesenchymal transition and tumor metastasis in lung cancer through modulating ERK1/2 and JAK2 signaling. Cell Death Dis. 2018;9:802.

20. Ding C, Tang W, Wu H, Fan X, Luo J, Feng J, et al. The PEAK1-PPP1R12B axis inhibits tumor growth and metastasis by regulating Grb2/PI3K/Akt signalling in colorectal cancer. Cancer Lett. 2019;442:383-395.

21. Huang L, Wen C, Yang X, Lou Q, Wang X, Che J, et al. PEAK1, acting as a tumor promoter in colorectal cancer, is regulated by the EGFR/KRas signaling axis and miR-181d. Cell Death Dis. 2018;9:271.

22. Yeh HW, Lee SS, Chang CY, Lang YD, Jou YS. A new switch for TGFbeta in cancer. Cancer Res. 2019;79:3797-3805.

23. Horton ER, Byron A, Askari JA, Ng DHJ, Millon-Fremillon A, Robertson J, et al. Definition of a consensus integrin adhesome and its dynamics during adhesion complex assembly and disassembly. Nat Cell Biol. 2015;17:1577-1587.

24. Chastney MR, Lawless C, Humphries JD, Warwood S, Jones MC, Knight D. Topological features of integrin adhesion complexes revealed by multiplexed proximity biotinylation. J Cell Biol. 2020;219:1-16. 8 e202003038.

25. Zheng Y, Zhang C, Croucher DR, Soliman MA, St-Denis N, Pasculescu A, et al. Temporal regulation of EGF signalling networks by the scaffold protein Shc1. Nature. 2013;499:166-171. 
26. Lin JR, Fallahi-Sichani M, Sorger PK. Highly multiplexed imaging of single cells using a high-throughput cyclic immunofluorescence method. Nat Commun. 2015;6:8390.

27. Gyorffy B, Lanczky A, Eklund AC, Denkert C, Budczies J, Li Q, et al. An online survival analysis tool to rapidly assess the effect of 22,277 genes on breast cancer prognosis using microarray data of 1,809 patients. Breast Cancer Res Treat. 2010;123:725-731.

28. Nagy A, Lanczky A, Menyhart O, Gyorffy B. Validation of miRNA prognostic power in hepatocellular carcinoma using expression data of independent datasets. Sci Rep. 2018;8:9227.

29. Finak G, Bertos N, Pepin F, Sadekova S, Souleimanova M, Zhao H, et al. Stromal gene expression predicts clinical outcome in breast cancer. Nat Med. 2008;14:518-527.

30. Tchou J, Kossenkov AV, Chang L, Satija C, Herlyn M, Showe LC, et al. Human breast cancer associated fibroblasts exhibit subtype specific gene expression profiles. BMC Med Genomics. 2012;5:39.

31. Zijlstra A, Lewis J, Degryse B, Stuhlmann H, Quigley JP. The inhibition of tumor cell intravasation and subsequent metastasis via regulation of in vivo tumor cell motility by the tetraspanin CD151. Cancer Cell. 2008;13:221-234.

32. Zijlstra A, Mellor R, Panzarella G, Aimes RT, Hooper JD, Marchenko ND, et al. A quantitative analysis of rate-limiting steps in the metastatic cascade using humanspecific real-time polymerase chain reaction. Cancer Res. 2002;62:7083-7092.

33. Fluegen G, Avivar-Valderas A, Wang Y, Padgen MR, Williams JK, Nobre AR, et al. Phenotypic heterogeneity of disseminated tumour cells is preset by primary tumour hypoxic microenvironments. Nat Cell Biol. 2017;19:120-132.

34. Stoletov K, Willetts L, Paproski RJ, Bond DJ, Raha S, Jovel J, et al. Quantitative in vivo whole genome motility screen reveals novel therapeutic targets to block cancer metastasis. Nat Commun. 2018;9:2343.

35. Pawlikowska P, Tayoun T, Oulhen M, Faugeroux V, Rouffiac V, Aberlenc A, et al. Exploitation of the chick embryo chorioallantoic membrane (CAM) as a platform for anti-metastatic drug testing. Sci Rep. 2020;10:16876.

36. Do MT, Chai TF, Casey PJ, Wang M. Isoprenylcysteine carboxylmethyltransferase function is essential for RAB4A-mediated integrin beta3 recycling, cell migration and cancer metastasis. Oncogene. 2017;36:5757-5767.

37. Pereira B, Chin SF, Rueda OM, Vollan HK, Provenzano E, Bardwell HA, et al. The somatic mutation profiles of 2,433 breast cancers refines their genomic and transcriptomic landscapes. Nat Commun. 2016;7:11479.

38. Kabraji S, Ni J, Lin NU, Xie S, Winer EP, Zhao JJ. Drug resistance in HER2-positive breast cancer brain metastases: blame the barrier or the brain? Clin Cancer Res. 2018;24:1795-1804.

39. Yang Z, Yang X, Xu S, Jin P, Li X, Wei X, et al. Reprogramming of stromal fibroblasts by SNAI2 contributes to tumor desmoplasia and ovarian cancer progression. Mol Cancer. 2017;16:163.

40. Bashari MH, Fan F, Vallet S, Sattler M, Arn M, Luckner-Minden C, et al. Mcl-1 confers protection of Her2-positive breast cancer cells to hypoxia: therapeutic implications. Breast Cancer Res. 2016;18:26.

41. El-Haibi CP, Bell GW, Zhang J, Collmann AY, Wood D, Scherber CM, et al. Critical role for lysyl oxidase in mesenchymal stem cell-driven breast cancer malignancy. Proc Natl Acad Sci USA. 2012;109:17460-17465.

42. Bartoschek M, Oskolkov N, Bocci M, Lovrot J, Larsson C, Sommarin M, et al. Spatially and functionally distinct subclasses of breast cancer-associated fibroblasts revealed by single cell RNA sequencing. Nat Commun. 2018;9:5150.

43. Wang H, Lapek J, Fujimura K, Strnadel J, Liu B, Gonzalez DJ, et al. Pseudopodiumenriched atypical kinase 1 mediates angiogenesis by modulating GATA2dependent VEGFR2 transcription. Cell Disco. 2018;4:26.

44. Smith JC, Hagemann A, Saka Y, Williams PH. Understanding how morphogens work. Philos Trans R Soc Lond B Biol Sci. 2008:363:1387-1392.

45. Landis MD, Seachrist DD, Montanez-Wiscovich ME, Danielpour D, Keri RA. Gene expression profiling of cancer progression reveals intrinsic regulation of transforming growth factor-beta signaling in ErbB2/Neu-induced tumors from transgenic mice. Oncogene. 2005;24:5173-5190.

46. Seachrist DD, Sizemore ST, Johnson E, Abdul-Karim FW, Weber Bonk KL, Keri RA Follistatin is a metastasis 'ressor in a mouse model of HER2-positive breast cancer. Breast Cancer Res. 2017;19:66.

\section{ACKNOWLEDGEMENTS}

We thank Dr. Joan Brugge for sponsorship of Dr. Jonathan Kelber's ASCB-MAC Visiting Professorship and helpful discussions during the experiment design and manuscript writing/revising phases. We thank Dr. Lesley Ellies for providing Py230 cells. We thank members of the Developmental Oncogene Laboratory at California State University Northridge for valuable feedback during manuscript preparation. This work was supported by California State University Northridge College of Science and Mathematics; Wellcome Centre for Cell-Matrix Research at the University of Manchester; Ludwig Center at Harvard Medical School; Sidney Stern Memorial Trust Sutter family; Aylozyan Family Foundation; NIH NCI grant R00CA222554 (to I.K.Z.); DOD grant W81XWH-14-1-0222 (to I.K.Z.); Cancer Research UK programme grant C13329/A21671 (to M.J.H.); NCI Cancer Center Support Grant 2P30CA016520 (to J.T.); Linda and Paul Richardson Breast Cancer Research Funds (to J.T.); NSF Grant DUE1842386 (to C.S./A.A. for support of T.N.); NIH NIGMS grant SC1GM121182 (to J.A.K.); ASCB MAC Visiting Professorship (to J.A.K.); and US-UK Fulbright-CRUK Scholar Award (to J.A.K.).

\section{COMPETING INTERESTS}

The authors declare no competing interests.

\section{ADDITIONAL INFORMATION}

Supplementary information The online version contains supplementary materia available at https://doi.org/10.1038/s41388-021-01906-2.

Correspondence and requests for materials should be addressed to J.A.K.

Reprints and permission information is available at http://www.nature.com/ reprints

Publisher's note Springer Nature remains neutral with regard to jurisdictional claims in published maps and institutional affiliations.

Open Access This article is licensed under a Creative Commons Attribution 4.0 International License, which permits use, sharing, adaptation, distribution and reproduction in any medium or format, as long as you give appropriate credit to the original author(s) and the source, provide a link to the Creative Commons license, and indicate if changes were made. The images or other third party material in this article are included in the article's Creative Commons license, unless indicated otherwise in a credit line to the material. If material is not included in the article's Creative Commons license and your intended use is not permitted by statutory regulation or exceeds the permitted use, you will need to obtain permission directly from the copyright holder. To view a copy of this license, visit http://creativecommons. org/licenses/by/4.0/.

(c) The Author(s) 2021 